\title{
Continuous entanglement renormalization on the circle
}

\author{
Ling-Yan Hung ${ }^{1,2,3, *}$ and Guifré Vidal ${ }^{4,5}$ \\ ${ }^{1}$ State Key Laboratory of Surface Physics, Fudan University, Shanghai 200433, China \\ ${ }^{2}$ Department of Physics and Center for Field Theory and Particle Physics, Fudan University, Shanghai 200433, China \\ ${ }^{3}$ Institute for Nanoelectronic Devices and Quantum Computing, Fudan University, Shanghai 200433, China \\ ${ }^{4}$ Sandbox@Alphabet, Mountain View, California 94043, USA \\ ${ }^{5}$ Perimeter Institute for Theoretical Physics, 31 Caroline Street North, Waterloo, Ontario, Canada N2L $2 Y 5$
}

(Received 8 February 2021; accepted 21 June 2021; published 15 October 2021)

\begin{abstract}
The continuous multiscale entanglement renormalization ansatz (cMERA) is a variational class of states for quantum fields. As originally formulated, the cMERA applies to infinite systems only. In this paper we generalize the cMERA formalism to a finite circle, which we achieve by wrapping the action of the so-called entangler around the circle. This allows us to transform a cMERA on the line into a cMERA on the circle. In addition, in the case of a Gaussian cMERA for noninteracting quantum fields, the method of images allows us to prove the following result: If on the line a cMERA state is a good approximation to a ground state of a local quantum field theory (QFT) Hamiltonian, then (under mild assumptions on their correlation functions) the resulting cMERA on a circle is also a good approximation to the ground state of the same local QFT Hamiltonian on the circle.
\end{abstract}

DOI: 10.1103/PhysRevResearch.3.043044

The multiscale entanglement renormalization ansatz (MERA) [1,2] is a tensor network used to efficiently represent a class of quantum many-body wave functions on the lattice and as the basis for nonperturbative, numerical approaches to strongly interacting quantum systems [1-8]. In contrast to quantum Monte Carlo methods, MERA algorithms are not affected by the so-called sign problem and can therefore be applied to, e.g., systems of interacting fermions and frustrated quantum magnets. Other applications include statistical mechanics [9], quantum gravity [10-14], cosmology [15-17], error correction [18,19], or machine learning [20-23].

In pioneering work [24], Haegeman, Osborne, Verschelde, and Verstraete proposed the continuous MERA (cMERA) for quantum field theories (QFTs), a generalization of MERA from the lattice to the continuum. These authors also showed, with explicit examples, that cMERA can accurately approximate the long-distance properties of ground states of noninteracting QFTs. The ultimate goal of the cMERA program is to become a nonperturbative approach to strongly interacting QFTs (see Refs. [25-28] for steps in this direction) and in this way mirror the success of MERA on the lattice. In the meantime, however, the Gaussian cMERA for noninteracting QFTs [24,29-32] has already proven its worth. Besides providing a valuable proof of concept that the lattice MERA formalism can be carried over to the continuum, Gaussian cMERA has been used as a toy model also in the context of the anti-de Sitter and conformal field theory (AdS-CFT)

\footnotetext{
*1yhung@fudan.edu.cn

Published by the American Physical Society under the terms of the Creative Commons Attribution 4.0 International license. Further distribution of this work must maintain attribution to the author(s) and the published article's title, journal citation, and DOI.
}

correspondence and quantum gravity [33-40]. Importantly, the Gaussian cMERA recasts key aspects of lattice MERAoriginally formulated in terms of networks of tensors and numerical optimizations - in the language of free quantum fields, which is familiar to a broader spectrum of theoretical physicists. For instance, the interpretation of the lattice ansatz as an entangling evolution in scale (as revisited below), or its connection to conformal data at a quantum critical point [29], become very clear in their continuum, free-field realizations. In this sense, Gaussian cMERA contributes significantly to the conceptual foundations of the MERA formalism.

In this paper we generalize the cMERA to finite systems. Specifically, given a cMERA on the infinite line, we explain how to build a cMERA on a finite circle. This is accomplished by modifying the action of the cMERA's entangler on the line so that it wraps around the circle. Importantly, in the Gaussian case, where our proposal amounts to using the method of images (Appendix C), we will show the following result. If the initial cMERA on the line is a good approximation to the ground state of a QFT, then (under simple assumptions on the correlation functions) the resulting cMERA on the circle is guaranteed to also be a good approximation to the ground state of the QFT on that circle. We illustrate our findings with a relativistic free boson in one dimension. However, these results can also be generalized to higher dimensions, to build, e.g., a cMERA on a cylinder or a torus (Appendix D). A related generalization to systems with open boundary conditions and/or defects has been recently proposed by Franco-Rubio [41].

cMERA on the line. Let us start by considering a bosonic quantum field on the line. It is characterized by field operators $\phi(x), \pi(x)$ with $x \in \mathbb{R}$ and canonical commutation relations $[\phi(x), \pi(y)]=i \delta(x-y)$. We denote their Fourier transforms by $\phi(k), \pi(k)$ (Appendix A). Following the original proposal of Ref. [24], a one-parameter family of cMERA states $\left|\Psi^{\Lambda}(s)\right\rangle$ 
is then defined as

$$
\left|\Psi^{\Lambda}(s)\right\rangle \equiv \exp [-i s(L+K)]|\Lambda\rangle .
$$

Here, $|\Lambda\rangle$ is an unentangled state characterized by

$$
\left(\sqrt{\frac{\Lambda}{2}} \phi(k)+\frac{i}{\sqrt{2 \Lambda}} \pi(k)\right)|\Lambda\rangle=0, \quad \forall k \in \mathbb{R},
$$

$L$ is the nonrelativistic rescaling operator

$$
L \equiv \frac{1}{2} \int_{\mathbb{R}} d k\left[\pi(-k)\left(k \partial_{k}+1 / 2\right) \phi(k)+\text { H.c. }\right],
$$

and $K$ is the quasilocal entangler, given by [42]

$$
\begin{aligned}
K & \equiv \frac{1}{2} \int_{\mathbb{R}_{2}} d x d y g(x-y)[\pi(x) \phi(y)+\phi(x) \pi(y)] \\
& =\frac{1}{2} \int_{\mathbb{R}} d k g(k)[\pi(-k) \phi(k)+\phi(-k) \pi(k)],
\end{aligned}
$$

where the entangling profile $g(x)$ is picked at $x=0$ and vanishes exponentially fast for large $|x| \gg 1 / \Lambda$, with $1 / \Lambda$ being a characteristic length scale \{e.g., $g(x) \sim e^{-\Lambda|x|}$ in Ref. [31]\}, and $g(k) \equiv \int_{\mathbb{R}} d x e^{-i k x} g(x)$ is its Fourier transform.

Rescaled picture. In this paper we use a different picture rescaled by the relativistic rescaling operator

$$
D \equiv \frac{1}{2} \int_{\mathbb{R}} d k\left[\pi(-k)\left(k \partial_{k}+1\right) \phi(k)+\text { H.c. }\right] .
$$

The cMERA state is then $\left|\tilde{\Psi}^{\Lambda}(s)\right\rangle \equiv e^{i s D}\left|\Psi^{\Lambda}(s)\right\rangle$, or

$$
\left|\tilde{\Psi}^{\Lambda}(s)\right\rangle=\mathcal{P} \exp \left(-i \int_{0}^{s} d s^{\prime} \tilde{K}\left(s^{\prime}\right)\right)|\Lambda\rangle,
$$

where $\mathcal{P} \exp$ is a path-ordered exponential and $\tilde{K}(s) \equiv$ $e^{i s D}(L+K-D) e^{-i s D}$ is a scale-dependent entangler with

$$
\begin{aligned}
\tilde{K}(s) & =\frac{1}{2} \int_{\mathbb{R}_{2}} d x d y \tilde{g}(s, x-y)[\pi(x) \phi(y)+\phi(x) \pi(y)] \\
& =\frac{1}{2} \int_{\mathbb{R}} d k \tilde{g}(s, k)[\pi(-k) \phi(k)+\phi(-k) \pi(k)],
\end{aligned}
$$

with $\quad \tilde{g}(s, x) \equiv e^{s} g\left(e^{s} x\right)-\delta(x) / 2 \quad$ and $\quad \tilde{g}(s, k)=g\left(e^{-s} k\right)-$ $1 / 2$. Equation (7) describes a so-called entangling evolution in scale. Starting from the unentangled state $|\Lambda\rangle$, we act with the entangler $\tilde{K}(s)$, which initially (that is, for $s=0$ ) introduces entanglement at scale $1 / \Lambda$. As the evolution progresses, the entangler's characteristic length decreases exponentially with growing $s$ as $e^{-s} / \Lambda$ (see Fig. 1) in such a way that entanglement is introduced at shorter and shorter distances. The resulting cMERA state $\left|\tilde{\Psi}^{\Lambda}(s)\right\rangle$ is thus entangled in the range of distances $\left[e^{-s} / \Lambda, 1 / \Lambda\right]$ [43]. Qualitatively, this entanglement structure is compatible with the ground state of a massive QFT with mass $m \sim \Lambda$ (or correlation length $1 / \Lambda$ ) and a UV cutoff at momentum $k \sim e^{s} \Lambda$ (or UV length $e^{-s} / \Lambda$ ).

Annihilation operators and correlation functions. Since the above cMERA is the result of evolving the state $|\Lambda\rangle$, which is Gaussian, by the entangler $\tilde{K}(s)$, which is quadratic, it follows that $\left|\tilde{\Psi}^{\Lambda}(s)\right\rangle$ is also Gaussian and can thus be characterized in terms of some complete set of annihilation operators $\tilde{b}^{\Lambda}(s, k)$, that is,

$$
\tilde{b}^{\Lambda}(s, k)\left|\tilde{\Psi}^{\Lambda}(s)\right\rangle=0, \quad \forall k \in \mathbb{R}
$$
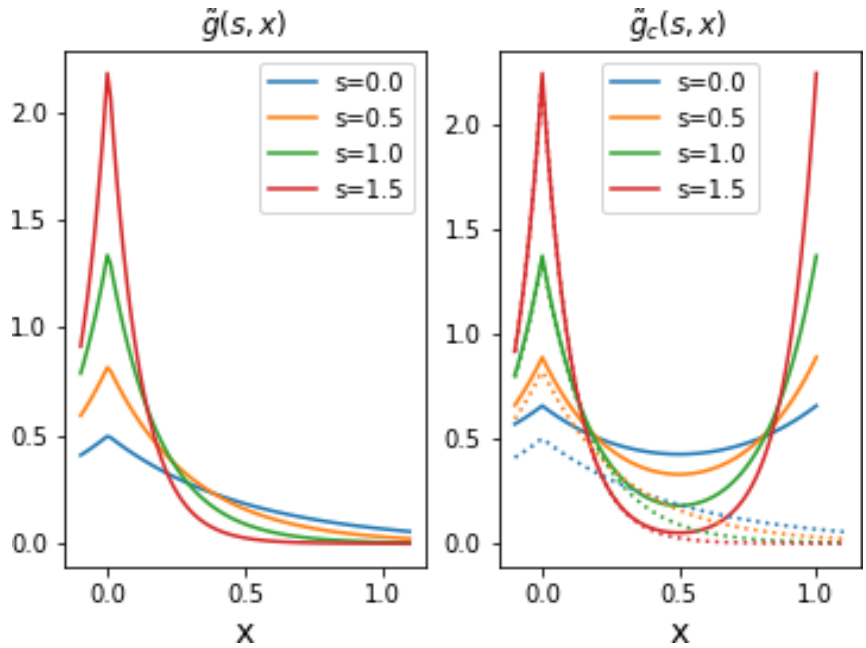

FIG. 1. Left: Example of entangling profile $\tilde{g}(s, x)$ on the line, which is picked at $x=0$ and has width $e^{-s} / \Lambda$ that decays exponentially with the scale parameter $s$. Right: Corresponding entangling profile $\tilde{g}_{c}(s, x)$ for a circle of size $l_{c}=1$, which is periodic by construction. These examples correspond to Eqs. (35) and (40) [excluding $-\delta(x) / 2]$, for $\Lambda=m=2$.

where we make the following ansatz:

$$
\tilde{b}^{\Lambda}(s, k) \equiv \sqrt{\frac{\tilde{\beta}(s, k)}{2}} \phi(k)+\frac{1}{\sqrt{2 \tilde{\beta}(s, k)}} \pi(k) .
$$

By imposing that $b^{\Lambda}(s, k)$ be the result of an entangling evolution in scale by $\tilde{K}(s)$ \{namely, $\frac{\partial}{\partial s} \tilde{b}^{\Lambda}(s, k)=$ $\left.-i\left[\tilde{K}(s), \tilde{b}^{\Lambda}(s, k)\right]\right\}$, we obtain the differential equation

$$
\frac{\partial}{\partial s} \tilde{\beta}(s, k)=-2 \tilde{g}(s, k) \tilde{\beta}(s, k), \quad \forall k \in \mathbb{R},
$$

relating $\tilde{g}(s, k)$ and $\tilde{\beta}(s, k)$, complemented by the initial condition $\tilde{\beta}(0, k)=\Lambda$, which enforces that the unentangled state $\left|\tilde{\Psi}^{\Lambda}(0)\right\rangle=|\Lambda\rangle$ is recovered at $s=0$; see Fig. 2. Finally, from Eq. (10) one obtains two-point correlation functions $C_{A B}(s, x-y) \equiv\left\langle\tilde{\Psi}^{\Lambda}(s)|A(x) B(y)| \tilde{\Psi}^{\Lambda}(s)\right\rangle$ (Appendix B),

$$
\begin{aligned}
& C_{\phi \phi}(s, x)=\int_{\mathbb{R}} \frac{d k}{2 \pi} e^{i k x} \frac{1}{2 \tilde{\beta}(s, k)}, \\
& C_{\pi \pi}(s, x)=\int_{\mathbb{R}} \frac{d k}{2 \pi} e^{i k x} \frac{\tilde{\beta}(s, k)}{2} .
\end{aligned}
$$

In summary, given an entangling profile $g(x)$ on the line, the resulting cMERA (including its correlation functions) is characterized by the solution $\widetilde{\beta}(s, k)$ of Eq. (12). Next we generalize this strategy to the circle.

cMERA on the circle. Consider a bosonic field on a circle of size $l_{c}$, with field operators $\phi(x), \pi(x)$ for $x \in\left[0, l_{c}\right)$ and $[\phi(x), \pi(y)]=i \delta(x-y)$. Their Fourier modes

$$
\begin{aligned}
& \phi(n) \equiv \frac{1}{\sqrt{l_{c}}} \int_{0}^{l_{c}} d x e^{-i k_{n} x} \phi(x), \\
& \pi(n) \equiv \frac{1}{\sqrt{l_{c}}} \int_{0}^{l_{c}} d x e^{-i k_{n} x} \pi(x)
\end{aligned}
$$



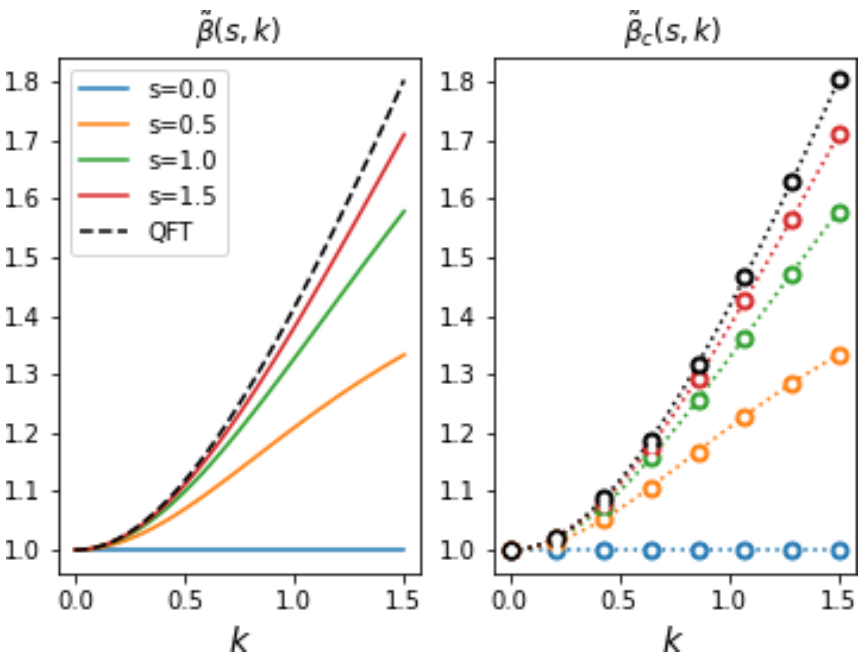

FIG. 2. Left: Function $\tilde{\beta}(s, k)$ characterizing the operators in Eqs. (10) and (11) that annihilate the cMERA state $\left|\tilde{\Psi}^{\Lambda}(s)\right\rangle$ on the line, for the specific choice in Eq. (37). For $s=0$ it produces the constant $\Lambda$ (corresponding to the unentangled state $|\Lambda\rangle$ ), whereas for large $s$ it tends to $\beta^{\mathrm{QFT}}(k)$ in Eq. (38) for a relativistic boson with mass $m=\Lambda=1$. Right: On a circle of length $l_{c}$, the method of images leads to a closely related function $\tilde{\beta}_{c}(s, n)$ that samples $\tilde{\beta}(s, k)$ at discrete momenta $k_{n}=2 \pi n / l_{c}$; see Eq. (26).

are now discrete, with $n \in \mathbb{Z}$ and $k_{n} \equiv 2 \pi n / l_{c}$. By analogy with (7), we define the cMERA state

$$
\left|\tilde{\Psi}_{c}^{\Lambda}(s)\right\rangle \equiv \mathcal{P} \exp \left(-i \int_{0}^{s} d s^{\prime} \tilde{K}_{c}\left(s^{\prime}\right)\right)\left|\Lambda_{c}\right\rangle .
$$

Here, $\left|\Lambda_{c}\right\rangle$ is the unentangled state characterized by

$$
\left(\sqrt{\frac{\Lambda}{2}} \phi(n)+\frac{i}{\sqrt{2 \Lambda}} \pi(n)\right)\left|\Lambda_{c}\right\rangle=0, \quad \forall n \in \mathbb{Z},
$$

and the scale-dependent entangler $\tilde{K}_{c}(s)$ is given by

$$
\begin{aligned}
\tilde{K}_{c}(s) & \equiv \frac{1}{2} \int_{0}^{l_{c}} d x d y \tilde{g}_{c}(s, x-y)[\pi(x) \phi(y)+\phi(x) \pi(y)] \\
& =\frac{1}{2} \sum_{n \in \mathbb{N}} \tilde{g}_{c}(s, n)[\pi(-n) \phi(n)+\phi(-n) \pi(n)],
\end{aligned}
$$

where $\tilde{g}_{c}(s, x)$ is some profile function and $\tilde{g}_{c}(s, n) \equiv$ $\int_{0}^{l_{c}} d x e^{-i k_{n} x} \tilde{g}_{c}(x)$ is its discrete Fourier transform.

Our key proposal is to build the profile function $\tilde{g}_{c}(s, x)$ on the circle from the profile function $\tilde{g}(s, x)$ on the line by adding contributions that come from wrapping the latter around the circle,

$$
\tilde{g}_{c}(s, x) \equiv \sum_{n \in \mathbb{Z}} \tilde{g}\left(s, x+n l_{c}\right) ;
$$

see Fig. 1. Equation (21) is seen to imply, through simple but tedious calculations (Appendixes $\mathrm{B}$ and $\mathrm{C}$ ), that most properties of the entangler and cMERA on the circle are closely related to those of the original constructions on the line. For starters, the Fourier transform $\tilde{g}_{c}(s, n)$ of $\tilde{g}_{c}(s, x)$ in (21) is given simply by (Appendix C)

$$
\tilde{g}_{c}(s, n)=\tilde{g}\left(s, k_{n}\right),
$$

that is, in terms of the function $\tilde{g}(s, k)$ on the line evaluated at discrete values $k=k_{n}$ of the momentum. The resulting Gaussian cMERA $\left|\tilde{\Psi}_{c}^{\Lambda}(s)\right\rangle$ is again characterized by a set of annihilation operators $b^{\Lambda}(s, n)$,

$$
\begin{gathered}
\tilde{b}^{\Lambda}(s, n)\left|\tilde{\Psi}_{c}^{\Lambda}(s)\right\rangle=0, \quad \forall n \in \mathbb{Z}, \\
\tilde{b}^{\Lambda}(s, n) \equiv \sqrt{\frac{\tilde{\beta}_{c}(s, n)}{2}} \phi(n)+\frac{1}{\sqrt{2 \tilde{\beta}_{c}(s, n)}} \pi(n),
\end{gathered}
$$

where coefficients $\tilde{\beta}_{c}(s, n)$ are determined by imposing $\frac{\partial}{\partial s} \tilde{b}^{\Lambda}(s, n)=-i\left[\tilde{K}_{c}(s), \tilde{b}^{\Lambda}(s, n)\right]$. This results in

$$
\frac{\partial}{\partial s} \tilde{\beta}_{c}(s, n)=-2 \tilde{g}_{c}(s, n) \tilde{\beta}_{c}(s, n), \quad \forall n \in \mathbb{Z},
$$

which is identical to Eq. (12) for $k=k_{n}$. Thus $\tilde{\beta}_{c}(s, n)$ on the circle is also given in terms of $\tilde{\beta}(s, k)$ on the line,

$$
\tilde{\beta}_{c}(s, n)=\tilde{\beta}\left(s, k_{n}\right) \text {; }
$$

see Fig. 2. Finally, from Eq. (23) we obtain correlation functions $C_{c, A B}(s, x-y) \equiv\left\langle\tilde{\Psi}_{c}^{\Lambda}(s)|A(x) B(y)| \tilde{\Psi}_{c}^{\Lambda}(s)\right\rangle$, which again relate simply to correlators on the line (Appendix C):

$$
\begin{aligned}
& C_{c, \phi \phi}(s, x)=\sum_{n \in \mathbb{Z}} C_{\phi \phi}\left(s, x+n l_{c}\right), \\
& C_{c, \pi \pi}(s, x)=\sum_{n \in \mathbb{Z}} C_{\pi \pi}\left(s, x+n l_{c}\right) .
\end{aligned}
$$

Therefore starting from a cMERA on the line, specified in terms of an entangling profile function $g(x)$, we have explained how to produce a closely related cMERA on the circle.

Suppose now that the cMERA state $\left|\tilde{\Psi}^{\Lambda}(s)\right\rangle$ approximates the ground state $\left|\Psi^{\mathrm{QFT}}\right\rangle$ of a noninteracting QFT on the line, in that the relative error $E_{A B}(s, x)$ between their correlators is at most some small $\epsilon(s)>0$, i.e.,

$$
E_{A B}(s, x) \equiv\left|\frac{C_{A B}(s, x)-C_{A B}^{\mathrm{QFT}}(x)}{C_{A B}^{\mathrm{QFT}}(x)}\right| \leqslant \epsilon(s),
$$

for all $x \geqslant x_{\mathrm{UV}}$, where $x_{\mathrm{UV}}$ is some UV length. Then, if $C_{A B}^{\mathrm{QFT}}(x)$ does not change sign for $x \geqslant x_{\mathrm{UV}}$, the proposed cMERA $\left|\tilde{\Psi}_{c}^{\Lambda}(s)\right\rangle$ on the circle also approximates the ground state $\left|\Psi_{c}^{\mathrm{QFT}}\right\rangle$ of the same QFT on the circle, in that, for all $x \geqslant x_{\mathrm{UV}}$, their correlators also fulfill

$$
E_{c, A B}(s, x) \equiv\left|\frac{C_{c, A B}(s, x)-C_{c, A B}^{\mathrm{QFT}}(x)}{C_{c, A B}^{\mathrm{QFT}}(x)}\right| \leqslant \epsilon(s) .
$$

This result (see Appendix B for a proof) follows quite simply from the fact that the QFT correlators on the line and on the circle obey relations analogous to Eqs. (27) and (28).

Example. Let $\left|\Psi^{\mathrm{QFT}}\right\rangle$ denote the ground state of the relativistic, free-boson Hamiltonian $H^{\mathrm{QFT}} \equiv \int_{\mathbb{R}} d x h(x)$ on the line, with local Hamiltonian density

$$
h(x) \equiv \frac{1}{2}\left[\pi(x)^{2}+\left(\partial_{x} \phi(x)\right)^{2}+m^{2} \phi(x)^{2}\right],
$$

where $m$ is the mass. As reviewed in Appendix A, $\left|\Psi^{\mathrm{QFT}}\right\rangle$ is annihilated by operators $b(k)$, i.e., $b(k)\left|\Psi^{\mathrm{QFT}}\right\rangle=0$, with

$$
b(k) \equiv \sqrt{\frac{\beta^{\mathrm{QFT}}(k)}{2}} \phi(k)+\frac{1}{\sqrt{2 \beta^{\mathrm{QFT}}(k)}} \pi(k),
$$



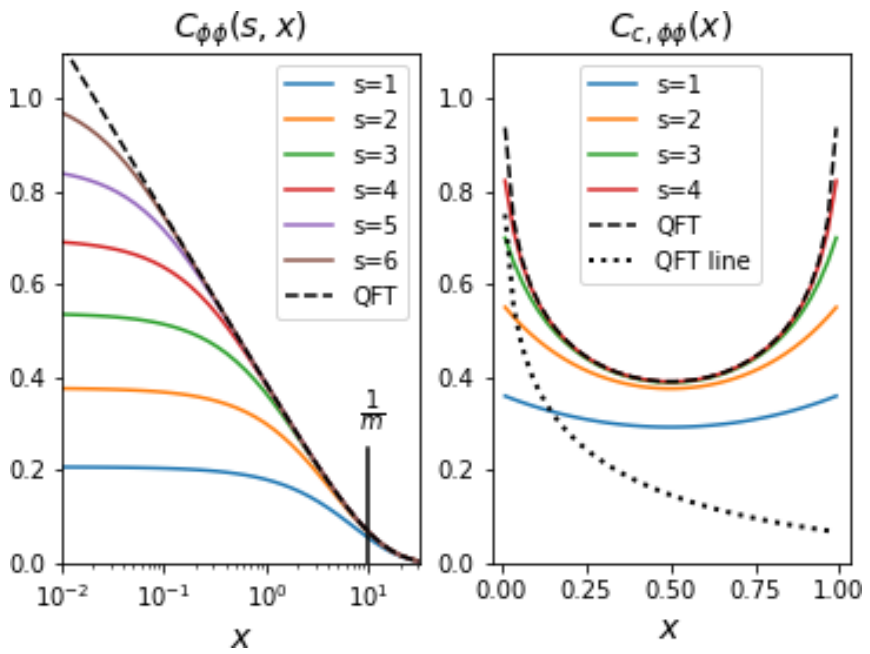

FIG. 3. Left: Correlation function $C_{\phi \phi}^{\mathrm{QFT}}(x)$ for the QFT ground state on the line with mass $m=0.1$ (dashed line) and $C_{\phi \phi}(s, x)$ for the corresponding cMERA with $\Lambda=m$. The cMERA correlator offers an accurate approximation for $x$ larger than $x_{\mathrm{UV}}=e^{-s} / \mathrm{m}$. Right: Same correlation functions on a circle of length $l_{c}=1$.

$$
\beta^{\mathrm{QFT}}(k) \equiv \sqrt{k^{2}+m^{2}} .
$$

This leads to correlation functions such as

$$
C_{\phi \phi}^{\mathrm{QFT}}(x)=K_{0}(m x) \sim \frac{1}{\sqrt{8 \pi}} \frac{e^{-m x}}{\sqrt{m x}},
$$

where $K_{0}$ is a modified Bessel function of the second kind and the expansion is for large distances $m x \gg 1$. Following Ref. [31], a cMERA approximation $\left|\tilde{\Psi}^{\Lambda}(s)\right\rangle$ is obtained with the so-called magic entangling profile $g(x)=\frac{\Lambda}{4} e^{-\Lambda|x|}$, which in the rescaled picture leads to

$$
\begin{gathered}
\tilde{g}(s, x)=\frac{e^{s} \Lambda}{4} e^{-e^{s} \Lambda|x|}-\frac{\delta(x)}{2}, \\
\tilde{g}(s, k)=\frac{\Lambda^{2}}{2\left(\Lambda^{2}+e^{2 s} k^{2}\right)}-\frac{1}{2} .
\end{gathered}
$$

Differential equation (12) is then solved by

$$
\tilde{\beta}(s, k)=\frac{\sqrt{k^{2}+\Lambda^{2}}}{\sqrt{1+\left(e^{-s} k / \Lambda\right)^{2}}},
$$

which indeed closely mimics $\beta^{\mathrm{QFT}}(k)$ for $\Lambda=m$,

$$
\tilde{\beta}(s, k)=\beta^{\mathrm{QFT}}(k)\left\{1+O\left[\left(\frac{e^{-s} k}{m}\right)^{2}\right]\right\} ;
$$

see Fig. 2. As a result, cMERA correlators accurately approximate QFT correlators; see Fig. 3 and Appendix B. For instance, for the $\phi \phi$ correlator we find

$$
C_{\phi \phi}(s, x)=C_{\phi \phi}^{\mathrm{QFT}}(x)\left[1+O\left(e^{-2 s}\right)\right], \quad m x \gg 1,
$$

so that the relative error between correlation functions is exponentially suppressed with $s, E_{\phi \phi}(s, x)=O\left(e^{-2 s}\right)$. Numerical evaluation shows that the error is small already for $x$ larger than the UV length $x_{\mathrm{UV}} \equiv e^{-s} / m$. Similar results are obtained for the $\pi \pi$ correlator.
Moving to a circle of size $l_{c}$, we consider the ground state $\left|\Psi_{c}^{\mathrm{QFT}}\right\rangle$ of $H_{c}^{\mathrm{QFT}}=\int_{0}^{l_{c}} d x h(x)$, where $h(x)$ is again the Hamiltonian density in Eq. (31). Its correlators are obtained by summing over images on the line, e.g., $C_{c, \phi \phi}^{\mathrm{QFT}}(x)=$ $\sum_{n} C_{\phi \phi}^{\mathrm{QFT}}\left(x+n l_{c}\right)$. Similarly, we build the cMERA's entangling profile $\tilde{g}_{c}(s, x)$ using Eq. (21). After explicitly summing over images we obtain (Appendix B)

$$
\tilde{g}_{c}(s, x)=\frac{e^{s} m}{4} \frac{\cosh \left[e^{s} m\left(\frac{l_{c}}{2}-x\right)\right]}{\sinh \left(e^{s} m \frac{l_{c}}{2}\right)}-\frac{\delta(x)}{2},
$$

which is shown in Fig. 1. The correlation functions of the resulting cMERA on the circle obey Eqs. (27) and (28). In particular, given that $C_{\phi \phi}^{\mathrm{QFT}}(x)$ on the line is positive for all $x>0$ (that is, it does not change sign), Eq. (39) implies that the relative error between ground-state and cMERA correlation functions on the circle is also exponentially suppressed with $s$ for $m x \gg 1$, namely, $E_{c, \phi \phi}(s, x)=O\left(e^{-2 s}\right)$. Numerical evaluation again shows that the error is small already for $x$ larger than the UV length $x_{\mathrm{UV}} \equiv e^{-s} / m$; see Fig. 3. Similar results are obtained for $\pi \pi$ correlation functions.

Discussion. In this paper we have extended the cMERA formalism from the infinite line to a finite circle, by wrapping the entangling profile $g(x)$ around the circle. This construction can be generalized straightforwardly to higher spatial dimensions, allowing one to also define the cMERA on, e.g., a cylinder or a torus (Appendix D). Notice that our proposal, discussed here for a Gaussian cMERA, is also valid for an interacting cMERA (with an entangler that includes terms that are, e.g., cubic or quartic in the fields). This is briefly discussed in Appendix D. However, only in the Gaussian case were we able to additionally show that, if the cMERA is a good approximation to a noninteracting QFT ground state on the line, then this property also holds on the circle. It is tempting to conclude by conjecturing that an equivalent result may still apply for an interacting cMERA, particularly when the theory is weakly interacting [44]. After all, that is known to be the case on the lattice. Indeed, a lattice MERA numerically optimized to resemble the ground state of an infinite spin chain is routinely used to build a MERA approximation for the ground state of a corresponding finite spin chain $[7,8,45]$.

L.-Y.H. acknowledges the support of NSFC (Grants No. 11922502 and No. 11875111) and the Shanghai Municipal Science and Technology Major Project (Shanghai Grant No. 2019SHZDZX01) and acknowledges Perimeter Institute for hospitality as a part of the Emmy Noether Fellowship Programme. G.V. acknowledges support as a CIFAR Fellow in the Quantum Information Science Program and a Distinguished Visiting Research Fellow at Perimeter Institute. Sandbox is a team within the Alphabet family of companies, which includes Google, Verily, Waymo, X, and others. Research at Perimeter Institute is supported by the Government of Canada through Innovation, Science and Economic Development Canada and by the Province of Ontario through the Ministry of Research, Innovation and Science. 


\section{APPENDIX A: ENTANGLING EVOLUTION ON THE LINE}

In this Appendix we derive in more detail the equations describing the entangling evolution in scale that generates the cMERA. Most of the material in this Appendix, concerned with the cMERA on the line, either already appeared scattered through previous work, mostly in Refs. [24,29-31], or could have been derived there with little effort. In particular, the accuracy analysis of the magic cMERA on the line, including short- and long-distance expansions, would have fitted naturally in Ref. [31]. Notice, however, that in contrast with Ref. [31], in this Appendix we work in the rescaled picture that we used in the main text, which is a most natural choice in order to subsequently analyze a circle of constant size $l_{c}$.

\section{1. cMERA on the line}

As in the main text, we consider a bosonic quantum field in one spatial dimension, as given by field operators $\phi(x)$ and $\pi(x)$ for $x \in \mathbb{R}$ with the commutation relation $[\phi(x), \pi(y)]=$ $i \delta(x-y)$. We also introduce Fourier transformed fields

$$
\begin{aligned}
& \phi(k) \equiv \frac{1}{\sqrt{2 \pi}} \int_{\mathbb{R}} d x e^{-i k x} \phi(x), \\
& \pi(k) \equiv \frac{1}{\sqrt{2 \pi}} \int_{\mathbb{R}} d x e^{-i k x} \pi(x),
\end{aligned}
$$

which obey $\phi(k)^{\dagger}=\phi(-k), \quad \pi(k)^{\dagger}=\pi(-k), \quad$ and $[\phi(k), \pi(q)]=i \delta(k+q)$. In terms of $\phi(k), \pi(k)$, the original fields $\phi(x), \pi(x)$ read

$$
\begin{aligned}
& \phi(x) \equiv \frac{1}{\sqrt{2 \pi}} \int_{\mathbb{R}} d k e^{i k x} \phi(k), \\
& \pi(x) \equiv \frac{1}{\sqrt{2 \pi}} \int_{\mathbb{R}} d k e^{i k x} \pi(k),
\end{aligned}
$$

where we have used

$$
\int_{\mathbb{R}} e^{i k x} d k=2 \pi \delta(x), \quad \int_{\mathbb{R}} e^{i k x} d x=2 \pi \delta(k) .
$$

Consider an arbitrary entangler

$$
\begin{aligned}
\tilde{K}(s)= & \frac{1}{2} \int_{\mathbb{R}_{2}} d x d y \tilde{g}(s, x-y)[\pi(x) \phi(y)+\phi(x) \pi(y)] \\
& =\frac{1}{2} \int_{\mathbb{R}} d k \tilde{g}(s, k)[\pi(-k) \phi(k)+\phi(-k) \pi(k)],
\end{aligned}
$$

where $\tilde{g}(s, x)$ is an entangling profile and $\tilde{g}(s, k) \equiv$ $\int_{\mathbb{R}} d x e^{-i k x} g(s, x)$ is its Fourier transform. We define the cMERA state $\left|\tilde{\Psi}^{\Lambda}(s)\right\rangle$ as an entangling evolution by $\tilde{K}(s)$,

$$
\left|\tilde{\Psi}^{\Lambda}(s)\right\rangle \equiv \mathcal{P} \exp \left(-i \int_{0}^{s} d s^{\prime} \tilde{K}\left(s^{\prime}\right)\right)|\Lambda\rangle,
$$

starting from the unentangled state $|\Lambda\rangle$, characterized by

$$
\begin{gathered}
\tilde{b}^{\Lambda}(0, k)|\Lambda\rangle=0, \quad \forall k \in \mathbb{R}, \\
\tilde{b}^{\Lambda}(0, k) \equiv \sqrt{\frac{\Lambda}{2}} \phi(k)+\frac{i}{\sqrt{2 \Lambda}} \pi(k) .
\end{gathered}
$$

In order to similarly characterize the cMERA state $\left|\tilde{\Psi}^{\Lambda}(s)\right\rangle$ in terms of a complete set of annihilation operators $\tilde{b}^{\Lambda}(s, k)$,

$$
\tilde{b}^{\Lambda}(s, k)\left|\tilde{\Psi}^{\Lambda}(s)\right\rangle=0, \quad \forall k \in \mathbb{R},
$$

we assume an expression of the form

$$
\tilde{b}^{\Lambda}(s, k) \equiv \sqrt{\frac{\tilde{\beta}(s, k)}{2}} \phi(k)+\frac{i}{\sqrt{2 \tilde{\beta}(s, k)}} \pi(k)
$$

and require that $\tilde{b}^{\Lambda}(s, k)$ change with $s$ according to the entangling evolution, that is,

$$
\frac{\partial}{\partial s} \tilde{b}^{\Lambda}(s, k)=-i\left[\tilde{K}(s), \tilde{b}^{\Lambda}(s, k)\right], \quad \forall k \in \mathbb{R},
$$

with Eq. (A10) as the initial conditions at $s=0$.

On the one hand, we have

$$
\begin{aligned}
& \frac{\partial}{\partial s} \tilde{b}^{\Lambda}(s, k) \\
& \quad=\frac{\partial}{\partial s}\left(\sqrt{\frac{\tilde{\beta}(s, k)}{2}} \phi(k)+\frac{i}{\sqrt{2 \tilde{\beta}(s, k)}} \pi(k)\right) \\
& \quad=\frac{\partial_{s} \tilde{\beta}(s, k)}{2 \tilde{\beta}(s, k)}\left(\sqrt{\frac{\tilde{\beta}(s, k)}{2}} \phi(k)-\frac{i}{\sqrt{2 \tilde{\beta}(s, k)}} \pi(k)\right) .
\end{aligned}
$$

On the other hand, the commutator reads

$$
\begin{aligned}
{\left[\tilde{K}(s), \tilde{b}^{\Lambda}(s, k)\right] } & =\frac{1}{2} \int_{\mathbb{R}} d q \tilde{g}(s, q)\left[\pi(-q) \phi(q)+\phi(-q) \pi(q), \sqrt{\frac{\tilde{\beta}(s, k)}{2}} \phi(k)+\frac{i \pi(k)}{\sqrt{2 \tilde{\beta}(s, k)}}\right] \\
& =-i \tilde{g}(s, k)\left(\sqrt{\frac{\tilde{\beta}(s, k)}{2}} \phi(k)-\frac{i}{\sqrt{2 \tilde{\beta}(s, k)}} \pi(k)\right) .
\end{aligned}
$$

Replacing these expressions in Eq. (A13), we conclude that $\tilde{\beta}(s, k)$ must obey the differential equation

$$
\frac{\partial}{\partial s} \tilde{\beta}(s, k)=-2 \tilde{g}(s, k) \tilde{\beta}(s, k), \quad \forall k \in \mathbb{R},
$$

with initial condition [cf. Eq. (A10)]

$$
\tilde{\beta}(s=0, k)=\Lambda, \quad \forall k \in \mathbb{R} .
$$


Its correlation functions in momentum space read

$$
\begin{gathered}
\left\langle\tilde{\Psi}^{\Lambda}(s)\left|\phi(k) \phi\left(k^{\prime}\right)\right| \tilde{\Psi}^{\Lambda}(s)\right\rangle \equiv \delta\left(k+k^{\prime}\right) \tilde{C}_{\phi \phi}^{\Lambda}(s, k), \\
\left\langle\tilde{\Psi}^{\Lambda}(s)\left|\pi(k) \pi\left(k^{\prime}\right)\right| \tilde{\Psi}^{\Lambda}(s)\right\rangle \equiv \delta\left(k+k^{\prime}\right) \tilde{C}_{\pi \pi}^{\Lambda}(s, k),
\end{gathered}
$$

for

$$
\tilde{C}_{\phi \phi}^{\Lambda}(s, k)=\frac{1}{2 \tilde{\beta}(s, k)}, \quad \tilde{C}_{\pi \pi}^{\Lambda}(s, k)=\frac{\tilde{\beta}(s, k)}{2},
$$

whereas in real space we have

$$
\begin{aligned}
\tilde{C}_{\phi \phi}^{\Lambda}(s, x) & \equiv\left\langle\tilde{\Psi}^{\Lambda}(s)|\phi(x) \phi(0)| \tilde{\Psi}^{\Lambda}(s)\right\rangle \\
& =\int_{\mathbb{R}} \frac{d k}{2 \pi} e^{i k x} \tilde{C}_{\phi \phi}^{\Lambda}(s, k), \\
\tilde{C}_{\pi \pi}^{\Lambda}(s, x) & \equiv\left\langle\tilde{\Psi}^{\Lambda}(s)|\pi(x) \pi(0)| \tilde{\Psi}^{\Lambda}(s)\right\rangle \\
& =\int_{\mathbb{R}} \frac{d k}{2 \pi} e^{i k x} \tilde{C}_{\pi \pi}^{\Lambda}(s, k) .
\end{aligned}
$$

\section{Comparison between pictures}

Consider a one-parameter family of cMERA states

$$
\left|\Psi^{\Lambda}(s)\right\rangle \equiv \exp [-i s(L+K)]|\Lambda\rangle,
$$

where $L$ is the nonrelativistic rescaling operator

$$
\begin{gathered}
L \equiv \frac{1}{2} \int_{\mathbb{R}} d k\left[\pi(-k)\left(k \partial_{k}+1 / 2\right) \phi(k)+\text { H.c. }\right], \\
e^{i s L} \phi(k) e^{-i s L}=e^{s / 2} \phi\left(e^{s} k\right), \\
e^{i s L} \pi(k) e^{-i s L}=e^{s / 2} \pi\left(e^{s} k\right),
\end{gathered}
$$

and $K$ is a quasilocal, scale-independent entangler

$$
\begin{aligned}
K & \equiv \frac{1}{2} \int_{\mathbb{R}_{2}} d x d y g(x-y)[\pi(x) \phi(y)+\phi(x) \pi(y)] \\
& =\frac{1}{2} \int_{\mathbb{R}} d k g(k)[\pi(-k) \phi(k)+\phi(-k) \pi(k)],
\end{aligned}
$$

which is expressed in terms of a scale-independent entangling profile $g(x)$. Here, $g(x)$ is equipped with a constant length scale $1 / \Lambda$. \{What is important at this stage is not that $K$ is independent of $s$, which we have chosen for simplicity, but rather that it entangles at a constant length scale $1 / \Lambda$. More generally, we could have chosen $g(s, x)$, leading to a scaledependent entangler $K(s)$ as in Ref. [24], still with constant entangling length $1 / \Lambda$.\}

In the main text we found it convenient to work instead with a rescaled picture in which the cMERA state $\left|\tilde{\Psi}^{\Lambda}(s)\right\rangle$ is given by

$$
\left|\tilde{\Psi}^{\Lambda}(s)\right\rangle \equiv e^{i s D}\left|\Psi^{\Lambda}(s)\right\rangle,
$$

where $D$ is the relativistic rescaling operator $D$,

$$
\begin{gathered}
D \equiv \frac{1}{2} \int_{\mathbb{R}} d k\left[\pi(-k)\left(k \partial_{k}+1\right) \phi(k)+\text { H.c. }\right], \\
e^{i s D} \phi(k) e^{-i s D}=e^{s} \phi\left(e^{s} k\right), \\
e^{i s D} \pi(k) e^{-i s D}=\pi\left(e^{s} k\right) .
\end{gathered}
$$

That leads to the expression in Eq. (A8) provided that the scale-dependent entangler $\tilde{K}(s)$ reads

$$
\begin{aligned}
\tilde{K}(s) \equiv & e^{i s D}(L+K-D) e^{-i s D} \\
= & \frac{1}{2} \int_{\mathbb{R}} d k\left(g(k)-\frac{1}{2}\right) \\
& \times e^{i s D}[\pi(-k) \phi(k)+\phi(-k) \pi(k)] e^{-i s D} \\
= & \frac{1}{2} \int_{\mathbb{R}} d k\left(g(k)-\frac{1}{2}\right) \\
& \times e^{s}\left[\pi\left(-e^{s} k\right) \phi\left(e^{s} k\right)+\phi\left(-e^{s} k\right) \pi\left(e^{s} k\right)\right] \\
= & \frac{1}{2} \int_{\mathbb{R}} d k\left(g\left(e^{-s} k\right)-\frac{1}{2}\right) \\
& \times[\pi(-k) \phi(k)+\phi(-k) \pi(k)],
\end{aligned}
$$

so that $\tilde{g}(s, k)=g\left(e^{-s} k\right)-1 / 2$.

\section{Relativistic free boson on the line}

The relativistic free boson has Hamiltonian

$$
H^{\mathrm{QFT}} \equiv \frac{1}{2} \int_{\mathbb{R}} d x\left\{\pi(x)^{2}+\left[\partial_{x} \phi(x)\right]^{2}+m^{2} \phi(x)^{2}\right\},
$$

where $m$ is the mass. We can diagonalize this Hamiltonian by first re-expressing it in terms of Fourier fields $\phi(k), \pi(k)$ and in terms of the annihilation operators $b(k)$, namely,

$$
\begin{aligned}
H^{\mathrm{QFT}} & =\frac{1}{2} \int_{\mathbb{R}} d k\left[\pi(-k) \pi(k)+\phi(-k)\left(k^{2}+m^{2}\right) \phi(k)\right] \\
& =\int_{\mathbb{R}} d k \sqrt{k^{2}+m^{2}} b(k)^{\dagger} b(k),
\end{aligned}
$$

with

$$
\begin{gathered}
b(k) \equiv \sqrt{\frac{\beta^{\mathrm{QFT}}(k)}{2}} \phi(k)+\frac{i}{\sqrt{2 \beta^{\mathrm{QFT}}(k)}} \pi(k), \\
\beta^{\mathrm{QFT}}(k) \equiv \sqrt{k^{2}+m^{2}} .
\end{gathered}
$$

Then its ground state $\left|\Psi^{\mathrm{QFT}}\right\rangle$ is characterized by being annihilated by $b(k)$,

$$
b(k)\left|\Psi^{\mathrm{QFT}}\right\rangle=0, \quad \forall k \in \mathbb{R} .
$$

Notice that $\left|\Psi^{\mathrm{QFT}}\right\rangle$ comes equipped with a momentum scale $m$. This is reflected in the correlation functions. In momentum space they read

$$
\begin{aligned}
& \left\langle\Psi^{\mathrm{QFT}}\left|\phi(k) \phi\left(k^{\prime}\right)\right| \Psi^{\mathrm{QFT}}\right\rangle \equiv \delta\left(k+k^{\prime}\right) C_{\phi \phi}^{\mathrm{QFT}}(k), \\
& \left\langle\Psi^{\mathrm{QFT}}\left|\pi(k) \pi\left(k^{\prime}\right)\right| \Psi^{\mathrm{QFT}}\right\rangle \equiv \delta\left(k+k^{\prime}\right) C_{\pi \pi}^{\mathrm{QFT}}(k),
\end{aligned}
$$

with

$$
C_{\phi \phi}^{\mathrm{QFT}}(k)=\frac{1}{2 \beta^{\mathrm{QFT}}(k)}, \quad C_{\pi \pi}^{\mathrm{QFT}}(k)=\frac{\beta^{\mathrm{QFT}}(k)}{2} .
$$

In real space we have

$$
\begin{aligned}
C_{\phi \phi}^{\mathrm{QFT}}(x) & \equiv\left\langle\Psi^{\mathrm{QFT}}|\phi(x) \phi(0)| \Psi^{\mathrm{QFT}}\right\rangle \\
& =\int_{\mathbb{R}} \frac{d k}{2 \pi} e^{i k x} C_{\phi \phi}^{\mathrm{QFT}}(k) \\
& =\frac{1}{2 \pi} K_{0}(m x)
\end{aligned}
$$


and

$$
\begin{aligned}
C_{\pi \pi}^{\mathrm{QFT}}(x) & \equiv\left\langle\Psi^{\mathrm{QFT}}|\pi(x) \pi(0)| \Psi_{m}^{\mathrm{QFT}}\right\rangle \\
& =\int_{\mathbb{R}} \frac{d k}{2 \pi} e^{i k x} C_{\pi \pi}^{\mathrm{QFT}}(k) \\
& =\frac{1}{2 \pi} \frac{m^{2}}{2}\left[K_{0}(m x)-K_{2}(m x)\right],
\end{aligned}
$$

where $K_{n}(x)$ is the modified Bessel function of the second kind and order $n$, with short-distance expansions

$$
K_{0}(x) \sim-\ln (x), \quad K_{2}(x) \sim \frac{1}{x^{2}},
$$

and long-distance expansion

$$
K_{n}(x) \sim \sqrt{\frac{\pi}{2}} \frac{e^{-x}}{\sqrt{x}}\left[1+\frac{4 n^{2}-1}{8 x}+O\left(\frac{1}{x^{2}}\right)\right] .
$$

We therefore find the short-distance expansions

$$
\begin{gathered}
C_{\phi \phi}^{\mathrm{QFT}}(x) \sim-\ln (m x), \\
C_{\pi \pi}^{\mathrm{QFT}}(x) \sim-\frac{1}{4 \pi} \frac{1}{x^{2}}
\end{gathered}
$$

for $m x \ll 1$, both of which diverge in the limit $m x \rightarrow 0$. For later reference, we add the correlator

$$
C_{\partial_{x} \phi \partial_{x} \phi}^{\mathrm{QFT}}(x) \sim \frac{1}{x^{2}},
$$

obtained by taking the second derivative of $C_{\phi \phi}^{\mathrm{QFT}}(x)$ with respect to $x$. We also have the long-distance expansions

$$
\begin{aligned}
C_{\phi \phi}^{\mathrm{QFT}}(x) & =\sqrt{\frac{\pi}{2}} \frac{e^{-m x}}{\sqrt{m x}}\left[1+O\left(\frac{1}{m x}\right)\right], \\
C_{\pi \pi}^{\mathrm{QFT}}(x) & =-\sqrt{\frac{\pi}{2}} \frac{e^{-m x}}{\sqrt{m x}}\left[1+O\left(\frac{1}{m x}\right)\right]
\end{aligned}
$$

for $m x \gg 1$, indicating that both correlation functions decay exponentially, with correlation length $1 / m$.

\section{Magic cMERA on the line}

In the main text we use the magic entangler from Ref. [31], given by

$$
g(k)=\frac{1}{2} \frac{\Lambda^{2}}{k^{2}+\Lambda^{2}}
$$

as an explicit example. It leads to

$$
\tilde{g}(s, k)=g\left(e^{-s} k\right)-\frac{1}{2}=-\frac{1}{2} \frac{\left(e^{-s} k\right)^{2}}{\left(e^{-s} k\right)^{2}+\Lambda^{2}} .
$$

Then, one can check that

$$
\tilde{\beta}(s, k)=\Lambda \sqrt{\frac{k^{2}+\Lambda^{2}}{\left(e^{-s} k\right)^{2}+\Lambda^{2}}}
$$

is a solution of the differential equation (C73) with the correct initial conditions $\tilde{\beta}(s=0, k)=\Lambda$. Notice that $\left|\tilde{\Psi}^{\Lambda}(s)\right\rangle$ comes equipped with two momentum scales: $\Lambda$ and $e^{s} \Lambda$, which we can think of as IR and UV scales, respectively. Specifically, its

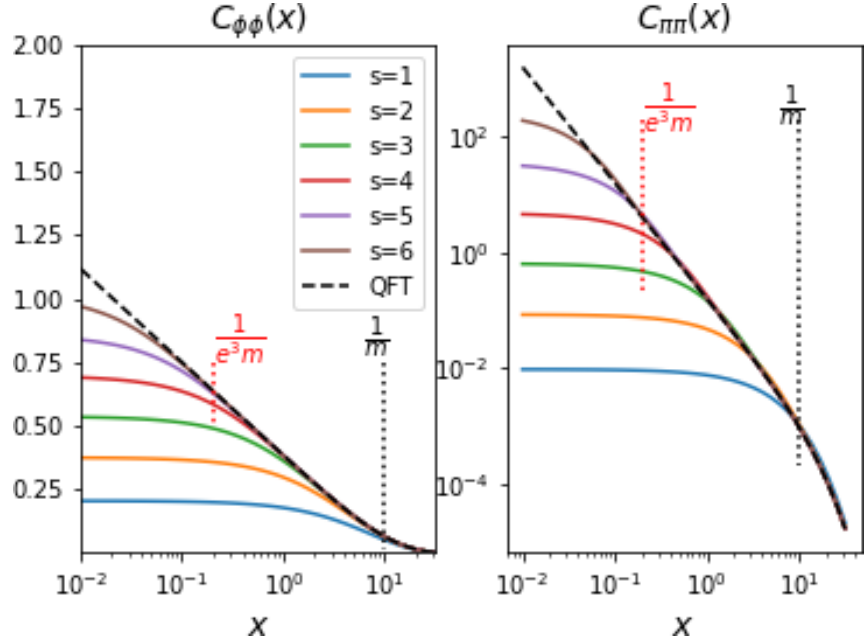

FIG. 4. Left: Real-space correlation functions $C_{\phi \phi}^{\mathrm{QFT}}(x)$ for the ground state of the relativistic free boson on the line with mass $m=0.1$ (dashed black line) and $\tilde{C}_{\phi \phi}^{\Lambda}(s, x)$ for the corresponding magic cMERA with $\Lambda=m$ and several values of the scale parameter $s$ (solid lines of varying color). Notice two characteristic lengths: the IR length $1 / m=10$, present both for the ground state of the relativistic free boson and the cMERA, and the UV length $1 /\left(e^{s} m\right)$, which is only present in the cMERA and decays exponentially in $s$. The ground-state correlator diverges as $x \rightarrow 0$, whereas the cMERA correlators tend to a finite constant for $x$ smaller than the UV length $e^{-s} / m$. Right: Same as left panel, but for the real-space correlation functions $C_{\pi \pi}^{\mathrm{QFT}}(x)$ for the ground state of the relativistic free boson and $\tilde{C}_{\pi \pi}^{\Lambda}(s, x)$ for the magic cMERA.

correlation functions in momentum space read

$$
\begin{gathered}
\left\langle\tilde{\Psi}^{\Lambda}(s)\left|\phi(k) \phi\left(k^{\prime}\right)\right| \tilde{\Psi}^{\Lambda}(s)\right\rangle \equiv \delta\left(k+k^{\prime}\right) \tilde{C}_{\phi \phi}^{\Lambda}(s, k), \\
\left\langle\tilde{\Psi}^{\Lambda}(s)\left|\pi(k) \pi\left(k^{\prime}\right)\right| \tilde{\Psi}^{\Lambda}(s)\right\rangle \equiv \delta\left(k+k^{\prime}\right) \tilde{C}_{\pi \pi}^{\Lambda}(s, k),
\end{gathered}
$$

for

$$
\tilde{C}_{\phi \phi}^{\Lambda}(s, k)=\frac{1}{2 \tilde{\beta}(s, k)}, \quad \tilde{C}_{\pi \pi}^{\Lambda}(s, k)=\frac{\tilde{\beta}(s, k)}{2},
$$

whereas in real space we have

$$
\begin{aligned}
\tilde{C}_{\phi \phi}^{\Lambda}(s, x) & \equiv\left\langle\tilde{\Psi}^{\Lambda}(s)|\phi(x) \phi(0)| \tilde{\Psi}^{\Lambda}(s)\right\rangle \\
& =\int_{\mathbb{R}} \frac{d k}{2 \pi} e^{i k x} \tilde{C}_{\phi \phi}^{\Lambda}(s, k), \\
\tilde{C}_{\pi \pi}^{\Lambda}(s, x) & \equiv\left\langle\tilde{\Psi}^{\Lambda}(s)|\pi(x) \pi(0)| \tilde{\Psi}^{\Lambda}(s)\right\rangle \\
& =\int_{\mathbb{R}} \frac{d k}{2 \pi} e^{i k x} \tilde{C}_{\pi \pi}^{\Lambda}(s, k) .
\end{aligned}
$$

Figure 4 shows the real-space correlation functions $\tilde{C}_{\phi \phi}^{\Lambda}(s, x)$ and $\tilde{C}_{\pi \pi}^{\Lambda}(s, x)$, obtained through a numerical Fourier transformation, for several values of the scale parameter $s$. One can see that these correlation functions behave differently in three regimes, separated by the UV length $e^{-s} / \Lambda$ and the IR length $1 / \Lambda$ : (i) For $x \in\left[0, e^{-s} / \Lambda\right]$, the correlator is approximately constant, with a value that grows with $s$; (ii) for $x \in\left[e^{-s} / \Lambda, 1 / \Lambda\right], \tilde{C}_{\phi \phi}^{\Lambda}(s, x)$ and $\tilde{C}_{\pi \pi}^{\Lambda}(s, x)$ scale approximately as $-\ln (\mu x)$ and $1 / x^{2}$, respectively; (iii) for $x>1 / \Lambda$, 
the correlation functions decay exponentially, with correlation length $1 / \Lambda$.

We remark that, had we not moved to the rescaled picture with $\left|\tilde{\Psi}^{\Lambda}(s)\right\rangle=e^{i s D}\left|\Psi^{\Lambda}(s)\right\rangle$ but used $\left|\Psi^{\Lambda}(s)\right\rangle$ instead, the corresponding correlation functions $C_{\phi \phi}^{\Lambda}(s, x)$ and $C_{\pi \pi}^{\Lambda}(s, x)$ would have had their UV and IR length scales be $1 / \Lambda$ (instead of $\left.e^{-s / \Lambda}\right)$ and $e^{s} / \Lambda$ (instead of $\left.1 / \Lambda\right)$, respectively.

\section{Ground state versus magic cMERA on the line}

We are now ready to compare the ground state $\left|\Psi^{\mathrm{QFT}}\right\rangle$ of the relativistic free boson with the magic cMERA $\left|\Psi^{\Lambda}(s)\right\rangle$ of Ref. [31]. They are annihilated, respectively, by operators $b(k)$ and $\tilde{b}^{\Lambda}(s, k)$, given by functions $\beta^{\mathrm{QFT}}(k)$ and $\tilde{\beta}(s, k)$ :

$$
\begin{gathered}
\beta^{\mathrm{QFT}}(k)=\sqrt{k^{2}+m^{2}} \\
\tilde{\beta}(s, k)=\sqrt{k^{2}+\Lambda^{2}} \frac{1}{\sqrt{\left(e^{-s} k / \Lambda\right)^{2}+1}} \\
=\sqrt{k^{2}+\Lambda^{2}}\left\{1+O\left[\left(\frac{e^{-s} k}{m}\right)^{2}\right]\right\} .
\end{gathered}
$$

We thus see that if we choose $\Lambda=m>0$ (in the massless case $m=0$, this requires introducing a small mass $m>0$ as an IR regulator), then we find that

$$
\begin{aligned}
\tilde{\beta}(s, k) & =\sqrt{k^{2}+m^{2}} \frac{1}{\sqrt{\left(e^{-s} k / m\right)^{2}+1}} \\
& =\beta^{\mathrm{QFT}}(k)\left\{1+O\left[\left(\frac{e^{-s} k}{m}\right)^{2}\right]\right\} .
\end{aligned}
$$

That is, the cMERA annihilation operators are similar to the ground-state annihilation operators for momenta $k$ smaller than the UV momentum $e^{s} m$. More specifically, Eqs. (A68) and (A76) together imply

$$
\begin{aligned}
& \tilde{C}_{\phi \phi}^{\Lambda}(s, k)=C_{\phi \phi}^{\mathrm{QFT}}(k)\left\{1+O\left[\left(\frac{e^{-s} k}{m}\right)^{2}\right]\right\}, \\
& \tilde{C}_{\pi \pi}^{\Lambda}(s, k)=C_{\pi \pi}^{\mathrm{QFT}}(k)\left\{1+O\left[\left(\frac{e^{-s} k}{m}\right)^{2}\right]\right\},
\end{aligned}
$$

indicating that the cMERA correlators match those of the QFT ground state at small momenta. Then, through a Fourier transform to real space we find

$$
\begin{aligned}
& \tilde{C}_{\phi \phi}^{\Lambda}(s, x)=C_{\phi \phi}^{\mathrm{QFT}}(x)+O\left(\frac{e^{-2 s}}{m^{2}} \frac{d^{2}}{d x^{2}} C_{\phi \phi}^{\mathrm{QFT}}(x)\right), \\
& \tilde{C}_{\pi \pi}^{\Lambda}(s, k)=C_{\pi \pi}^{\mathrm{QFT}}(x)+O\left(\frac{e^{-2 s}}{m^{2}} \frac{d^{2}}{d x^{2}} C_{\pi \pi}^{\mathrm{QFT}}(x)\right) .
\end{aligned}
$$

Next we consider two regimes for which we can derive approximate expressions.

Firstly, for $m x \ll 1$, but assuming $x \gg e^{-s} / m$ (that is, $k \ll$ $e^{s} m$ ), from the short-distance expansions (A58) and (A59), we obtain

$$
\frac{d^{2}}{d x^{2}} C_{\phi \phi}^{\mathrm{QFT}}(x) \sim \frac{1}{x^{2}}, \quad \frac{d^{2}}{d x^{2}} C_{\pi \pi}^{\mathrm{QFT}}(x) \sim \frac{1}{x^{4}},
$$

and therefore

$$
\begin{gathered}
\tilde{C}_{\phi \phi}^{\Lambda}(s, x)=C_{\phi \phi}^{\mathrm{QFT}}(x)\left[1+O\left(\frac{e^{-2 s}}{(m x)^{2} \ln (m x)}\right)\right], \\
\tilde{C}_{\pi \pi}^{\Lambda}(s, k)=C_{\pi \pi}^{\mathrm{QFT}}(x)\left[1+O\left(\frac{e^{-2 s}}{(m x)^{2}}\right)\right] .
\end{gathered}
$$

Similarly, we would find

$$
\tilde{C}_{\partial_{x} \phi \partial_{x} \phi}^{\Lambda}(s, k)=C_{\partial_{x} \phi \partial_{x} \phi}^{\mathrm{QFT}}(x)\left[1+O\left(\frac{e^{-2 s}}{(m x)^{2}}\right)\right] .
$$

Recall that this is the setting relevant to the massless case, $m=0$, where we introduce a small mass $m$ as an IR regulator. Thus the cMERA succeeds at recovering the power-law decay [or, for $C_{\phi \phi}^{\mathrm{QFT}}(x)$, the logarithmic scaling] characteristic of the correlation functions of the free-boson conformal field theory, provided that $x \in\left[e^{-s} / m, 1 / m\right]$ is not too close to the UV length $e^{-s} / m$ and IR length $1 / m$.

Secondly, for $x \gg 1 / m$, Eqs. (A61) and (A62) imply

$$
\begin{aligned}
& \frac{d^{2}}{d x^{2}} C_{\phi \phi}^{\mathrm{QFT}}(x)=m^{2} C_{\phi \phi}^{\mathrm{QFT}}(x)\left[1+O\left(\frac{1}{m x}\right)\right], \\
& \frac{d^{2}}{d x^{2}} C_{\pi \pi}^{\mathrm{QFT}}(x)=m^{2} C_{\pi \pi}^{\mathrm{QFT}}(x)\left[1+O\left(\frac{1}{m x}\right)\right],
\end{aligned}
$$

and therefore

$$
\begin{aligned}
& \tilde{C}_{\phi \phi}^{\Lambda}(s, x)=C_{\phi \phi}^{\mathrm{QFT}}(x)\left[1+O\left(e^{-2 s}\right)\right], \\
& \tilde{C}_{\pi \pi}^{\Lambda}(s, x)=C_{\pi \pi}^{\mathrm{QFT}}(x)\left[1+O\left(e^{-2 s}\right)\right] .
\end{aligned}
$$

Expressions (A82)-(A84), (A87), and (A88) will be useful in the next Appendix, when trying to characterize the accuracy of cMERA for the ground state of the relativistic boson on the circle.

Notice that (A83), (A84), (A87), and (A88) imply that, for $x$ in the relevant regime of values, the relative error between cMERA and ground-state correlators on the line is exponentially suppressed with $s$,

$$
\begin{aligned}
& E_{\phi \phi}(x) \equiv\left|\frac{\tilde{C}_{\phi \phi}^{\Lambda}(s, k)-C_{\phi \phi}^{\mathrm{QFT}}(x)}{C_{\phi \phi}^{\mathrm{QFT}}(x)}\right|=O\left(e^{-2 s}\right), \\
& E_{\pi \pi}(x) \equiv\left|\frac{\tilde{C}_{\pi \pi}^{\Lambda}(s, k)-C_{\pi \pi}^{\mathrm{QFT}}(x)}{C_{\pi \pi}^{\mathrm{QFT}}(x)}\right|=O\left(e^{-2 s}\right) .
\end{aligned}
$$

Finally, the insets in Figs. 5 and 6 show the actual errors $E_{\phi \phi}(s, x)$ and $E_{\pi \pi}(s, x)$ computed numerically.

\section{APPENDIX B: ENTANGLING EVOLUTION ON THE CIRCLE}

In this Appendix we provide a more detailed derivation of several of the expressions for the cMERA on the circle that were used in the main text.

\section{1. cMERA on the circle}

We consider a bosonic field on a circle of size $l_{c}$, with field operators $\phi(x), \pi(x)$ for $x \in\left[0, l_{c}\right)$ and canonical com- 


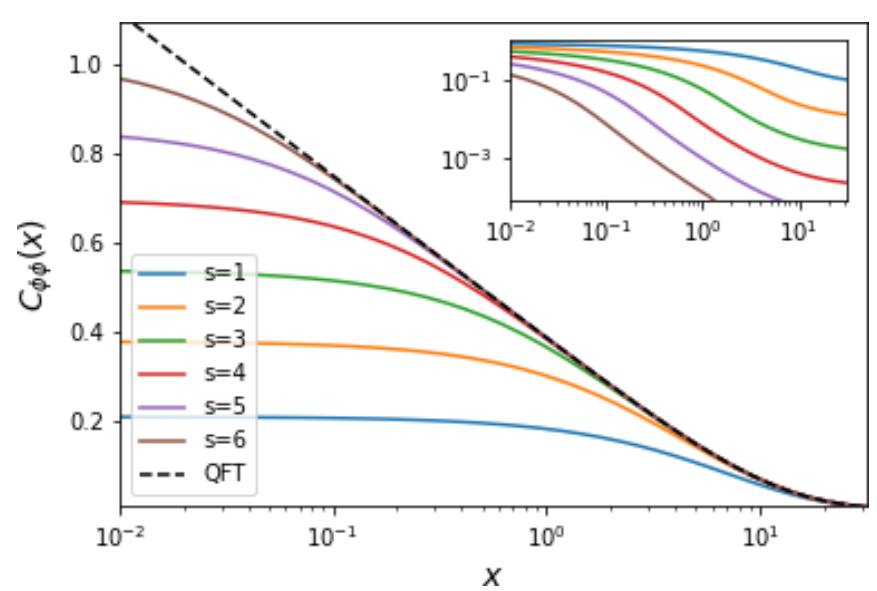

FIG. 5. Same as the left panel of Fig. 4. The inset shows the relative error $E_{\phi \phi}(s, x)$ between the ground-state and cMERA correlators, which starts to decay sharply with $x$ for values of $x$ larger than the UV length $x_{\mathrm{Uv}} e^{-s} / m$.

mutation relations $[\phi(x), \pi(y)]=i \delta(x-y)$. We introduce a discrete set of Fourier modes

$$
\begin{aligned}
& \phi(n) \equiv \frac{1}{\sqrt{l_{c}}} \int_{0}^{l_{c}} d x e^{-i k_{n} x} \phi(x), \\
& \pi(n) \equiv \frac{1}{\sqrt{l_{c}}} \int_{0}^{l_{c}} d x e^{-i k_{n} x} \pi(x),
\end{aligned}
$$

with $n \in \mathbb{Z}$ and $k_{n} \equiv 2 \pi n / l_{c}$. They obey $\phi(n)^{\dagger}=\phi(-n)$, $\pi(n)^{\dagger}=\pi(-n)$ and commutation relations $[\phi(n), \pi(n)]=$ $i \delta_{n,-m}$. In terms of $\phi(n), \pi(n)$, the original fields $\phi(x), \pi(x)$ read

$$
\begin{aligned}
& \phi(x)=\frac{1}{\sqrt{l_{c}}} \sum_{n} e^{i k_{n} x} \phi(n), \\
& \pi(x)=\frac{1}{\sqrt{l_{c}}} \sum_{n} e^{i k_{n} x} \pi(n),
\end{aligned}
$$

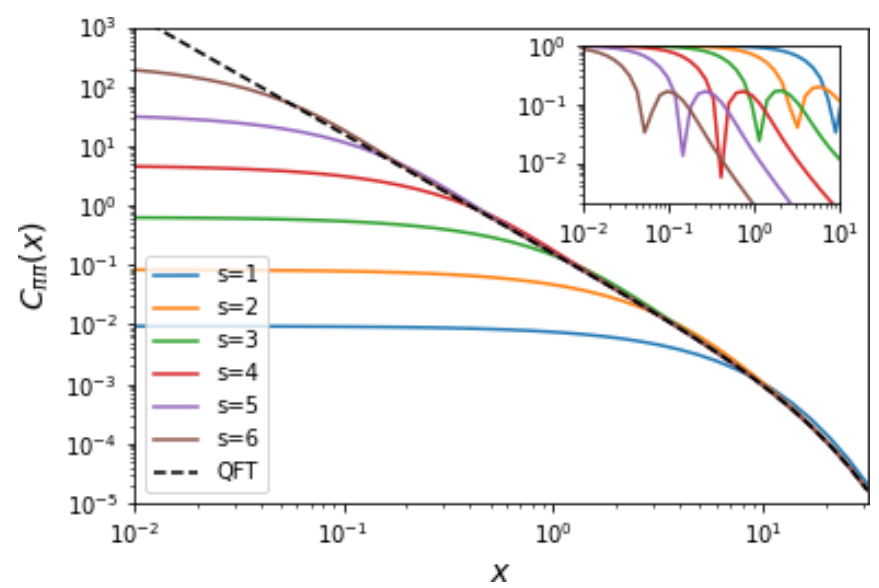

FIG. 6. Same as the right panel of Fig. 4. The inset shows the relative error $E_{\pi \pi}(s, x)$ between the ground-state and cMERA correlators, which starts to decay sharply with $x$ for values of $x$ larger than the UV length $x_{\mathrm{Uv}} e^{-s} / m$. where we have used

$$
\begin{aligned}
& \int_{0}^{l_{c}} e^{i\left(k_{n}-k_{m}\right) x} d x=l_{c} \delta_{n, m}, \\
& \sum_{n \in \mathbb{Z}} e^{i k_{n}(x-y)}=l_{c} \delta(x-y),
\end{aligned}
$$

where the last expression follows from $\sum_{n} e^{i a x n}=\frac{2 \pi}{a} \delta(x)$.

Consider an entangler $\tilde{K}_{c}(s)$ of the form

$$
\begin{gathered}
\tilde{K}_{c}(s)=\frac{1}{2} \int_{0}^{l_{c}} d x d y \tilde{g}_{c}(s, x-y)[\pi(x) \phi(y)+\phi(x) \pi(y)] \\
=\frac{1}{2} \sum_{n \in \mathbb{N}} \tilde{g}_{c}(s, n)[\pi(-n) \phi(n)+\phi(-n) \pi(n)],
\end{gathered}
$$

where $\tilde{g}_{c}(s, x)$ is an entangling profile and $\tilde{g}_{c}(s, n) \equiv$ $\int_{0}^{l_{c}} d x e^{-i k_{n} x} \tilde{g}_{c}(s, x)$ is its discrete Fourier transform. We define the cMERA state $\left|\tilde{\Psi}_{c}^{\Lambda}(s)\right\rangle$ as an entangling evolution by $\tilde{K}_{c}(s)$,

$$
\left|\tilde{\Psi}_{c}^{\Lambda}(s)\right\rangle \equiv \mathcal{P} \exp \left(-i \int_{0}^{s} d s^{\prime} \tilde{K}_{c}\left(s^{\prime}\right)\right)\left|\Lambda_{c}\right\rangle,
$$

starting from the unentangled state $\left|\Lambda_{c}\right\rangle$ given by

$$
\begin{gathered}
\tilde{b}^{\Lambda}(0, n)\left|\Lambda_{c}\right\rangle=0, \quad \forall n \in \mathbb{Z}, \\
\tilde{b}^{\Lambda}(0, n) \equiv \sqrt{\frac{\Lambda}{2}} \phi(n)+\frac{i}{\sqrt{2 \Lambda}} \pi(n) .
\end{gathered}
$$

In order to characterize the cMERA state $\left|\tilde{\Psi}_{c}^{\Lambda}(s)\right\rangle$ in terms of a complete set of annihilation operators $\tilde{b}^{\Lambda}(s, n)$,

$$
\tilde{b}^{\Lambda}(s, n)\left|\Psi_{c}^{\Lambda}(s)\right\rangle=0, \quad \forall n \in \mathbb{Z},
$$

we assume an expression of the form

$$
\tilde{b}^{\Lambda}(s, n) \equiv \sqrt{\frac{\tilde{\beta}_{c}(s, n)}{2}} \phi(n)+\frac{i}{\sqrt{2 \tilde{\beta}_{c}(s, n)}} \pi(n)
$$

and require that the operators $\tilde{b}^{\Lambda}(s, n)$ change with $s$ according to the entangling evolution, that is,

$$
\frac{\partial}{\partial s} \tilde{b}^{\Lambda}(s, n)=-i\left[\tilde{K}_{c}(s), \tilde{b}^{\Lambda}(s, n)\right], \quad \forall n \in \mathbb{Z},
$$

with Eq. (B11) as the initial conditions at $s=0$. Notice that Eq. (B14) is formally equivalent to Eq. (A13). Following the derivation after Eq. (A13), we conclude that $\tilde{\beta}_{c}(s, n)$ must obey the differential equation

$$
\frac{\partial}{\partial s} \tilde{\beta}_{c}(s, n)=-2 \tilde{g}_{c}(s, n) \tilde{\beta}_{c}(s, n), \quad \forall n \in \mathbb{Z},
$$

with initial condition [cf. Eq. (B11)]

$$
\tilde{\beta}_{c}(s=0, n)=\Lambda, \quad \forall n \in \mathbb{Z} .
$$

\section{Relativistic free boson on the circle}

The relativistic free boson on a circle has Hamiltonian

$$
H_{c}^{\mathrm{QFT}} \equiv \frac{1}{2} \int_{0}^{l_{c}} d x\left\{\pi(x)^{2}+\left[\partial_{x} \phi(x)\right]^{2}+m^{2} \phi(x)^{2}\right\},
$$


where $m$ is the mass and $l_{c}$ is the circle's perimeter. As on the line, we diagonalize $H_{c}^{\mathrm{QFT}}$ by first re-expressing it in terms of Fourier fields $\phi(n), \pi(n)$ and then in terms of the annihilation operators $b_{m}(n)$, namely,

$$
\begin{aligned}
H_{c}^{\mathrm{QFT}} & =\frac{1}{2} \sum_{n \in \mathbb{Z}}\left[\pi(-n) \pi(n)+\phi(-n)\left(k_{n}^{2}+m^{2}\right) \phi(n)\right] \\
& =\sum_{n \in \mathbb{Z}} \sqrt{k_{n}^{2}+m^{2}} b(n)^{\dagger} b(n),
\end{aligned}
$$

with $k_{n} \equiv 2 \pi n / l_{c}$ and

$$
\begin{gathered}
b(n) \equiv \sqrt{\frac{\beta_{c}^{\mathrm{QFT}}(n)}{2}} \phi(n)+\frac{i}{\sqrt{2 \beta_{c}^{\mathrm{QFT}}(n)}} \pi(n), \\
\beta_{c}^{\mathrm{QFT}}(n) \equiv \sqrt{k_{n}^{2}+m^{2}} .
\end{gathered}
$$

Then its ground state $\left|\Psi_{c}^{\mathrm{QFT}}\right\rangle$ is characterized by being annihilated by $b(n)$,

$$
b(n)\left|\Psi_{c}^{\mathrm{QFT}}\right\rangle=0, \quad \forall n \in \mathbb{Z} .
$$

The correlators in momentum space read

$$
\begin{aligned}
& \left\langle\Psi_{c}^{\mathrm{QFT}}\left|\phi(n) \phi\left(n^{\prime}\right)\right| \Psi_{c}^{\mathrm{QFT}}\right\rangle \equiv \delta_{n,-n^{\prime}} C_{c, \phi \phi}^{\mathrm{QFT}}(n), \\
& \left\langle\Psi_{c}^{\mathrm{QFT}}\left|\pi(n) \pi\left(n^{\prime}\right)\right| \Psi_{c}^{\mathrm{QFT}}\right\rangle \equiv \delta_{n,-n^{\prime}} C_{c, \pi \pi}^{\mathrm{QFT}}(n),
\end{aligned}
$$

with

$$
C_{c, \phi \phi}^{\mathrm{QFT}}(n)=\frac{1}{2 \beta_{c}^{\mathrm{QFT}}(n)}, \quad C_{c, \pi \pi}^{\mathrm{QFT}}(n)=\frac{\beta_{c}^{\mathrm{QFT}}(n)}{2}
$$

and, in real space,

$$
\begin{aligned}
C_{c, \phi \phi}^{\mathrm{QFT}}(x) & \equiv\left\langle\Psi_{c}^{\mathrm{QFT}}|\phi(x) \phi(0)| \Psi_{c}^{\mathrm{QFT}}\right\rangle \\
& =\frac{1}{l_{c}} \sum_{n \in \mathbb{Z}} e^{i k_{n} x} C_{c, \phi \phi}^{\mathrm{QFT}}(n), \\
C_{c, \pi \pi}^{\mathrm{QFT}}(x) & \equiv\left\langle\Psi_{c}^{\mathrm{QFT}}|\phi(x) \phi(0)| \Psi_{c}^{\mathrm{QFT}}\right\rangle \\
& =\frac{1}{l_{c}} \sum_{n \in \mathbb{Z}} e^{i k_{n} x} C_{c, \pi \pi}^{\mathrm{QFT}}(n) .
\end{aligned}
$$

Using the method of images (reviewed in Appendix C), we can then relate the real-space correlators on the circle to those on the line through

$$
\begin{aligned}
& C_{c, \phi \phi}^{\mathrm{QFT}}(x)=\sum_{n \in \mathbb{Z}} C_{\phi \phi}^{\mathrm{QFT}}\left(x+n l_{c}\right), \\
& C_{c, \pi \pi}^{\mathrm{QFT}}(x)=\sum_{n \in \mathbb{Z}} C_{\pi \pi}^{\mathrm{QFT}}\left(x+n l_{c}\right)
\end{aligned}
$$

and thus express them in terms of the explicit solutions [modified Bessel functions of the second kind $K_{0}(m x)$ and $K_{2}(m x)$; see Eqs. (A52) and (A55)] and corresponding short-distance and long-distance expansions we found on the line.

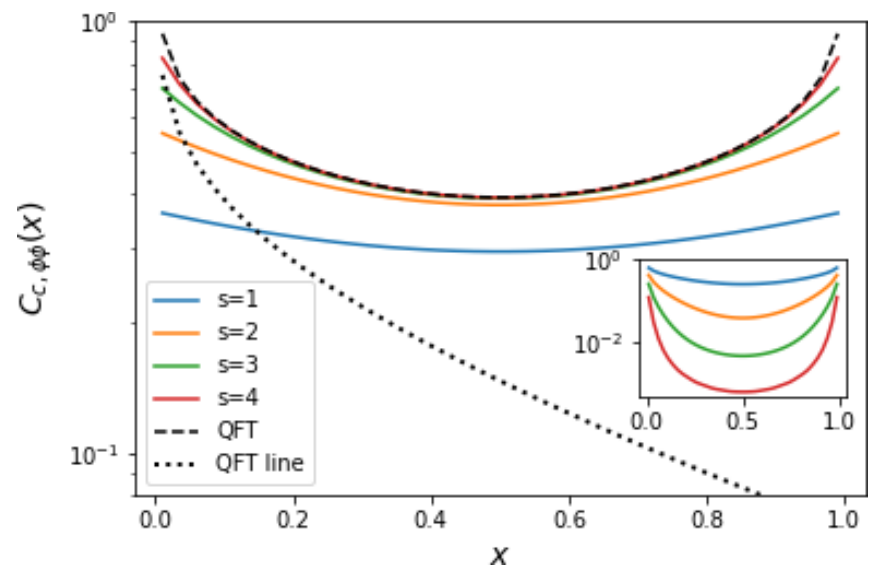

FIG. 7. Real-space correlation functions $C_{c, \phi \phi}^{\mathrm{QFT}}(x)$ for the ground state of the relativistic free boson with mass $m=1$ on a circle of length $l_{c}=1$ (dashed black line; the correlation function on the line is shown as a dotted black line) and $\tilde{C}_{c, \phi \phi}^{\Lambda}(s, x)$ for the corresponding magic cMERA with $\Lambda=m$ and several values of the scale parameter $s$ (solid lines of varying color). The inset shows the relative error $E_{c, \phi \phi}(s, x)$ between the ground-state and cMERA correlators, which starts to decay sharply with $x$ for values of $x$ larger than the UV length $x_{\mathrm{Uv}} e^{-s} / m$.

In particular, if we consider the short-distance expansion (valid for $m x \ll 1$ ) and artificially expand it to arbitrary values of $x \in \mathbb{R}_{+}$, we obtain

$$
\begin{gathered}
C_{c, \phi \phi}^{\mathrm{QFT}}(x) \sim-\sum_{n \in \mathbb{Z}} \ln \left(m\left|x+n l_{c}\right|\right), \\
C_{c, \pi \pi}^{\mathrm{QFT}}(x) \sim-\sum_{n \in \mathbb{Z}} \frac{1}{\left(x+n l_{c}\right)^{2}} .
\end{gathered}
$$

The sum for $C_{c, \phi \phi}^{\mathrm{QFT}}(x)$ diverges for all $x>0$, but the sum for $C_{c, \pi \pi}^{\mathrm{QFT}}(x)$ is finite, as is also the (equivalent) sum for $C_{c, \partial_{x} \phi \partial_{x} \phi}^{\mathrm{QFT}}(x)$; see Eq. (A60). This is consistent with the wellknown fact that, in the free-boson CFT, the field $\phi(x)$ is not necessarily a well-defined object, but the fields $\pi(x)$ and $\partial_{x} \phi(x)$ are.

On the other hand, if we assume that the circle is large compared with the correlation length (that is, $m l_{c} \gg 1$ ) and consider a value of $x \in\left[0, l_{c}\right]$ such that the long-distance expansion is valid (that is, $m\left|x+n l_{c}\right| \gg 1$ ), we obtain for both $C_{c, \phi \phi}^{\mathrm{QFT}}(x)$ and $C_{c, \pi \pi}^{\mathrm{QFT}}(x)$ a sum of the form

$$
\sqrt{\frac{\pi}{2}} \sum_{n \in \mathbb{Z}} \frac{e^{-m\left|x+n l_{c}\right|}}{\sqrt{m\left|x+n l_{c}\right|}}\left[1+O\left(\frac{1}{m\left|x+n l_{c}\right|}\right)\right],
$$

where the terms in the sum are exponentially suppressed with growing values of $|n|$ and therefore converge. See Figs. 7 and 8 .

\section{Example: Magic cMERA on the circle}

In the main text we have adjusted the magic entangler of Ref. [31] (originally proposed for the line) to the circle, with 


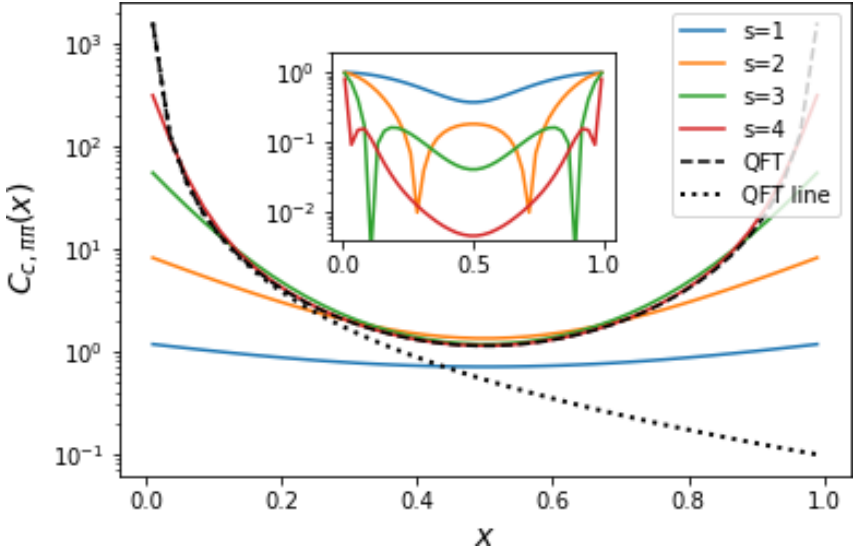

FIG. 8. Real-space correlation functions $C_{c, \pi \pi}^{\mathrm{QFT}}(x)$ for the ground state of the relativistic free boson with mass $m=1$ on a circle of length $l_{c}=1$ (dashed black line; the correlation function on the line is shown as a dotted black line) and $\tilde{C}_{c, \pi \pi}^{\Lambda}(s, x)$ for the corresponding magic cMERA with $\Lambda=m$ and several values of the scale parameter $s$ (solid lines of varying color). The inset shows the relative error $E_{c, \pi \pi}(s, x)$ between the ground-state and cMERA correlators, which starts to decay sharply with $x$ for values of $x$ larger than the UV length $x_{\mathrm{Uv}} e^{-s} / m$.

real-space entangling profile

$$
\begin{aligned}
g_{c}(s, x) & \equiv \frac{e^{s} \Lambda}{4} \sum_{n \in \mathbb{Z}} e^{-e^{s} \Lambda\left|x+n l_{c}\right|} \\
& =\frac{e^{s} \Lambda}{4} \frac{\cosh \left[e^{s} \Lambda\left(\frac{l_{c}}{2}-x\right)\right]}{\sinh \left(e^{s} \Lambda \frac{l_{c}}{2}\right)},
\end{aligned}
$$

where we used that, for $a \in[0, b]$,

$$
\begin{aligned}
\sum_{n \in \mathbb{Z}} e^{-|a+n b|} & =\left(e^{-a}+e^{-(b-a)}\right) \sum_{n=0}^{\infty} e^{-n b} \\
& =\left(e^{\frac{b}{2}-a}+e^{-\left(\frac{b}{2}-a\right)}\right) \frac{e^{-\frac{b}{2}}}{1-e^{-b}} \\
& =\frac{\cosh \left(\frac{b}{2}-a\right)}{\sinh \left(\frac{b}{2}\right)}
\end{aligned}
$$

and momentum space profile

$$
\tilde{g}_{c}(s, n)=-\frac{1}{2} \frac{\left(e^{-s} k_{n}\right)^{2}}{\left(e^{-s} k_{n}\right)^{2}+\Lambda^{2}} .
$$

This results in the solution $\tilde{\beta}_{c}(s, n)$ of the differential equation (B15) given by

$$
\tilde{\beta}_{c}(s, n)=\Lambda \sqrt{\frac{k_{n}^{2}+\Lambda^{2}}{\left(e^{-s} k_{n}\right)^{2}+\Lambda^{2}}},
$$

which indeed fulfills the initial condition (B11). The resulting cMERA has correlators

$$
\begin{aligned}
\left\langle\tilde{\Psi}_{c}^{\Lambda}(s)\left|\phi(n) \phi\left(n^{\prime}\right)\right| \tilde{\Psi}_{c}^{\Lambda}(s)\right\rangle & =\delta_{n,-n^{\prime}} \tilde{C}_{c, \phi \phi}^{\Lambda}(s, n), \\
\left\langle\tilde{\Psi}_{c}^{\Lambda}(s)\left|\pi(n) \pi\left(n^{\prime}\right)\right| \tilde{\Psi}_{c}^{\Lambda}(s)\right\rangle & =\delta_{n,-n^{\prime}} \tilde{C}_{c, \pi \pi}^{\Lambda}(s, n),
\end{aligned}
$$

for

$$
\tilde{C}_{c, \phi \phi}^{\Lambda}(s, n)=\frac{1}{2 \tilde{\beta}_{c}(s, n)}, \quad \tilde{C}_{c, \pi \pi}^{\Lambda}(s, n)=\frac{\tilde{\beta}_{c}(s, n)}{2},
$$

or, in real space,

$$
\begin{aligned}
\tilde{C}_{c, \phi \phi}^{\Lambda}(s, x) & \equiv\left\langle\tilde{\Psi}_{c}^{\Lambda}(s)|\phi(x) \phi(0)| \tilde{\Psi}_{c}^{\Lambda}(s)\right\rangle \\
& =\frac{1}{l_{c}} \sum_{n \in \mathbb{Z}} e^{i k_{n} x} \tilde{C}_{c, \phi \phi}^{\Lambda}(n), \\
\tilde{C}_{c, \pi \pi}^{\Lambda}(s, x) & \equiv\left\langle\tilde{\Psi}_{c}^{\Lambda}(s)|\phi(x) \phi(0)| \tilde{\Psi}_{c}^{\Lambda}(s)\right\rangle \\
& =\frac{1}{l_{c}} \sum_{n \in \mathbb{Z}} e^{i k_{n} x} \tilde{C}_{c, \pi \pi}^{\Lambda}(n) .
\end{aligned}
$$

Using the method of images (reviewed in Appendix C), we can also relate the real-space correlators on the circle to those on the line through

$$
\begin{aligned}
& \tilde{C}_{c, \phi \phi}^{\Lambda}(s, x)=\sum_{n \in \mathbb{Z}} \tilde{C}_{\phi \phi}^{\Lambda}\left(s, x+n l_{c}\right), \\
& \tilde{C}_{c, \pi \pi}^{\Lambda}(s, x)=\sum_{n \in \mathbb{Z}} \tilde{C}_{\pi \pi}^{\Lambda}\left(s, x+n l_{c}\right) .
\end{aligned}
$$

\section{Ground state versus magic cMERA on the circle}

The comparison between the ground state $\left|\Psi^{\mathrm{QFT}}\right\rangle$ of the relativistic free boson and the magic cMERA $\left|\tilde{\Psi}_{c}^{\Lambda}(s)\right\rangle$ on the circle can be conducted, thanks to the method of images, by simply translating expressions from the line to the circle. In particular, combining Eqs. (A79) and (A80) with Eqs. (B49) and (B50), we readily obtain

$$
\begin{aligned}
& \tilde{C}_{c, \phi \phi}^{\Lambda}(s, x)=C_{c, \phi \phi}^{\mathrm{QFT}}(x)+O\left(\frac{e^{-2 s}}{m^{2}} \frac{d^{2}}{d x^{2}} C_{c, \phi \phi}^{\mathrm{QFT}}(x)\right), \\
& \tilde{C}_{c, \pi \pi}^{\Lambda}(s, k)=C_{c, \pi \pi}^{\mathrm{QFT}}(x)+O\left(\frac{e^{-2 s}}{m^{2}} \frac{d^{2}}{d x^{2}} C_{c, \pi \pi}^{\mathrm{QFT}}(x)\right) .
\end{aligned}
$$

Then, using the $m x \ll 1$ expansion (for the massless case, with $m>0$ being an IR regulator to be set to zero), we find that Eqs. (A83) and (A84) imply that

$$
\begin{gathered}
\tilde{C}_{c, \pi \pi}^{\Lambda}(s, k)=C_{c, \pi \pi}^{\mathrm{QFT}}(x)\left[1+O\left(e^{-2 s}\right)\right], \\
\tilde{C}_{c, \partial_{x} \phi \partial_{x} \phi}^{\Lambda}(s, k)=C_{c, \partial_{x} \phi \partial_{x} \phi}^{\mathrm{QFT}}(x)\left[1+O\left(e^{-2 s}\right)\right] .
\end{gathered}
$$

Similarly, for $m l_{c} \gg 1$ (circle of large length compared with correlation length), for $x \in\left[0, l_{c}\right]$ such that the long-distance expansion is valid (that is, $m\left|x+n l_{c}\right| \gg 1$ ), Eqs. (A87) and (A88) imply

$$
\begin{aligned}
& \tilde{C}_{c, \phi \phi}^{\Lambda}(s, k)=C_{c, \phi \phi}^{\mathrm{QFT}}(x)\left[1+O\left(e^{-2 s}\right)\right], \\
& \tilde{C}_{c, \pi \pi}^{\Lambda}(s, k)=C_{c, \pi \pi}^{\mathrm{QFT}}(x)\left[1+O\left(e^{-2 s}\right)\right] .
\end{aligned}
$$

The above expressions imply that, for $x$ in the relevant regime of values, the relative error between cMERA and ground-state correlators on the circle is exponentially sup- 
pressed with $s$, e.g.,

$$
\begin{aligned}
& E_{c, \phi \phi}(s, x) \equiv\left|\frac{\tilde{C}_{c, \phi \phi}^{\Lambda}(s, x)-C_{c, \phi \phi}^{\mathrm{QFT}}(x)}{C_{c, \phi \phi}^{\mathrm{QFT}}(x)}\right|=O\left(e^{-2 s}\right), \\
& E_{c, \pi \pi}(s, x) \equiv\left|\frac{\tilde{C}_{c, \pi \pi}^{\Lambda}(s, x)-C_{c, \pi \pi}^{\mathrm{QFT}}(x)}{C_{c, \pi \pi}^{\mathrm{QFT}}(x)}\right|=O\left(e^{-2 s}\right) .
\end{aligned}
$$

More generally, consider a cMERA correlator $C^{\Lambda}(x)$ that approximates a QFT ground-state correlator $C^{\mathrm{QFT}}(x)$ on the line at distances larger than $x_{\mathrm{UV}}$, in the sense that there is a small $\epsilon>0$ such that for any $|x| \geqslant x_{\mathrm{UV}}$,

$$
\left|\frac{C^{\Lambda}(x)-C^{\mathrm{QFT}}(x)}{C^{\mathrm{QFT}}(x)}\right| \leqslant \epsilon .
$$

We will also assume that the QFT correlator does not change sign for $x \geqslant x_{\mathrm{UV}}$, say, $C^{\mathrm{QFT}}(x) \geqslant 0$ for all $x \geqslant x_{\mathrm{UV}}$. Consider now a cMERA and ground state on a circle of size $l_{c}$ obtained by the method of images (see Appendix C), so that their correlators $C_{c}^{\Lambda}(x)$ and $C_{c}^{\mathrm{QFT}}(x)$ read

$$
\begin{aligned}
C_{c}^{\Lambda}(x) & =\sum_{n \in \mathbb{Z}} C^{\Lambda}\left(x+n l_{c}\right), \\
C_{c}^{\mathrm{QFT}}(x) & =\sum_{n \in \mathbb{Z}} C^{\mathrm{QFT}}\left(x+n l_{c}\right) .
\end{aligned}
$$

Then, for any $x \in\left[x_{\mathrm{UV}}, l_{c} / 2\right]$ (that is, such that $\left|x+n l_{c}\right| \geqslant$ $\left.x_{\mathrm{UV}}\right)$ we have that also

$$
\left|\frac{C_{c}^{\Lambda}(x)-C_{c}^{\mathrm{QFT}}(x)}{C_{c}^{\mathrm{QFT}}(x)}\right| \leqslant \epsilon,
$$

so that also on the circle the cMERA correlator approximates the ground-state correlator.

Indeed, we have that

$$
\begin{aligned}
& \left|C_{c}^{\Lambda}(x)-C_{c}^{\mathrm{QFT}}(x)\right| \\
& =\left|\sum_{n \in \mathbb{Z}} C^{\Lambda}\left(x+n l_{c}\right)-C^{\mathrm{QFT}}\left(x+n l_{c}\right)\right| \\
& \leqslant \sum_{n \in \mathbb{Z}}\left|C^{\Lambda}\left(x+n l_{c}\right)-C^{\mathrm{QFT}}\left(x+n l_{c}\right)\right| \\
& \quad \leqslant \sum_{n \in \mathbb{Z}} \epsilon\left|C^{\mathrm{QFT}}\left(x+n l_{c}\right)\right| \\
& \quad=\epsilon\left|\sum_{n \in \mathbb{Z}} C^{\mathrm{QFT}}\left(x+n l_{c}\right)\right| \\
& \quad=\epsilon\left|C_{c}^{\mathrm{QFT}}(x)\right|,
\end{aligned}
$$

where in the last step we used that, by assumption, $C^{\mathrm{QFT}}(x+$ $n l_{c}$ ) does not change sign as a function of $n$.

\section{APPENDIX C: THE METHOD OF IMAGES}

In this Appendix we review the method of images, specialized to connecting the Fourier transforms of two closely related functions $f(x)$ and $f_{c}(x)$, one on the line and the other on the circle. First we state the main result and provide a proof.
Then we review in detail how this applies to several examples of interest in the main text.

\section{Relating the line and the circle}

Let us consider a function $f(x)$ defined on the real line, with $f(k)$ being its Fourier transform,

$$
\begin{gathered}
f(k) \equiv \int_{\mathbb{R}} d x e^{-i k x} f(x), \\
f(x)=\frac{1}{2 \pi} \int_{\mathbb{R}} d k e^{i k x} f(k) .
\end{gathered}
$$

Let us also consider a function $f_{c}(x)$ on a circle of size $l_{c}$, with $f_{c}(n)$ being its discrete Fourier transform,

$$
\begin{aligned}
f_{c}(n) & \equiv \int_{0}^{l_{c}} d x e^{-i k_{n} x} f_{c}(x), \\
f_{c}(x) & =\frac{1}{l_{c}} \sum_{n \in \mathbb{Z}} e^{i k_{n} x} f_{c}(n),
\end{aligned}
$$

where $k_{n} \equiv 2 \pi n / l_{c}$.

We will assume that $f(x)$ decays sufficiently fast with $|x|$ for large $|x|$, so that for any $x \in\left[0, l_{c}\right)$, the sum $\sum_{n \in \mathbb{Z}} f(x+$ $\left.n l_{c}\right)$ is finite.

Theorem (method of images).

$$
\begin{gathered}
f_{c}(x)=\sum_{n \in \mathbb{Z}} f\left(x+n l_{c}\right), \quad \forall x \in\left[0, l_{c}\right) \\
\Longleftrightarrow f_{c}(n)=f\left(k_{n}\right), \quad \forall n \in \mathbb{N} .
\end{gathered}
$$

In words, the above theorem says the following: $(\Rightarrow)$ If for all $x$ the function $f_{c}(x)$ on the circle can be obtained in terms of the function $f(x)$ on the real line by adding contributions from the points $x, x \pm l_{c}, x \pm 2 l_{c}$, etc., then the Fourier transform $f_{c}(n)$ on the circle can be obtained from the Fourier transform $f(k)$ on the line evaluated at momentum $k=k_{n}$. The theorem further establishes that the converse $(\Leftarrow)$ also holds. Namely, if the Fourier transform $f_{c}(n)$ on the circle samples the Fourier transform $f(k)$ on the line at momentum $k=k_{n}$, then the function $f_{c}(x)$ on the circle can be expressed as the above sum of contributions of the function $f(x)$ on the real line.

Proof. For $(\Rightarrow)$, we have

$$
\begin{aligned}
f_{c}(n) & =\int_{0}^{l_{c}} d x e^{-i k_{n} x} f_{c}(x) \\
& =\int_{0}^{l_{c}} d x e^{-i k_{n} x} \sum_{n \in \mathbb{Z}} f\left(x+n l_{c}\right) \\
& =\int_{0}^{l_{c}} d x e^{-i k_{n} x} \sum_{n \in \mathbb{Z}} \int_{\mathbb{R}} \frac{d k}{2 \pi} e^{i k\left(x+n l_{c}\right)} f(k) \\
& =\int_{0}^{l_{c}} d x e^{-i k_{n} x} \int_{\mathbb{R}} \frac{d k}{2 \pi} e^{i k x} f(k) \sum_{n \in \mathbb{Z}} e^{i k n l_{c}} \\
& =\frac{2 \pi}{l_{c}} \int_{0}^{l_{c}} d x e^{-i k_{n} x} \int_{\mathbb{R}} \frac{d k}{2 \pi} e^{i k x} f(k) \sum_{m \in \mathbb{Z}} \delta\left(k-k_{m}\right) \\
& =\frac{1}{l_{c}} \int_{0}^{l_{c}} d x e^{-i k_{n} x} \sum_{m \in \mathbb{Z}} e^{i k_{m} x} f\left(k_{m}\right)
\end{aligned}
$$




$$
\begin{aligned}
& =\sum_{m \in \mathbb{Z}} f\left(k_{m}\right) \frac{1}{l_{c}} \int_{0}^{l_{c}} d x e^{i\left(k_{m}-k_{n}\right) x} \\
& =\sum_{m \in \mathbb{Z}} f\left(k_{m}\right) \delta_{m, n} \\
& =f\left(k_{n}\right) .
\end{aligned}
$$

Here, we used that

$$
\sum_{n \in \mathbb{Z}} e^{i a n}=2 \pi \sum_{m \in \mathbb{Z}} \delta(a-2 \pi m),
$$

which implies

$$
\begin{aligned}
\sum_{n \in \mathbb{Z}} e^{i k n l_{c}} & =2 \pi \sum_{m \in \mathbb{Z}} \delta\left(k l_{c}-2 \pi m\right) \\
& =\frac{2 \pi}{l_{c}} \sum_{m \in \mathbb{Z}} \delta\left(k-\frac{2 \pi}{l_{c}} m\right) \\
& =\frac{2 \pi}{l_{c}} \sum_{m \in \mathbb{Z}} \delta\left(k-k_{m}\right) .
\end{aligned}
$$

For the converse $(\Leftarrow)$ we have

$$
\begin{aligned}
f_{c}(x) & =\frac{1}{l_{c}} \sum_{n \in \mathbb{Z}} e^{i k_{n} x} f_{c}(n) \\
& =\frac{1}{l_{c}} \sum_{n \in \mathbb{Z}} e^{i k_{n} x} f\left(k_{n}\right) \\
& =\frac{1}{l_{c}} \sum_{n \in \mathbb{Z}} e^{i k_{n} x} \int_{\mathbb{R}} d y e^{-i k_{n} y} f(y) \\
& =\int_{\mathbb{R}} d y f(y) \frac{1}{l_{c}} \sum_{n \in \mathbb{Z}} e^{i k_{n}(x-y)} \\
& =\int_{\mathbb{R}} d y f(y) \sum_{n \in \mathbb{Z}} \delta\left(x-y+n l_{c}\right) \\
& =\sum_{n \in \mathbb{Z}} f\left(x+n l_{c}\right),
\end{aligned}
$$

where we used that

$$
\begin{aligned}
\sum_{n \in \mathbb{Z}} e^{i k_{n} x} & =\sum_{n \in \mathbb{Z}} e^{i \frac{2 \pi}{l_{c}} x n} \\
& =2 \pi \sum_{n \in \mathbb{Z}} \delta\left(x \frac{2 \pi}{l_{c}}-2 \pi n\right) \\
& =l_{c} \sum_{n \in \mathbb{Z}} \delta\left(x-n l_{c}\right) .
\end{aligned}
$$

This completes the proof of the above theorem. Next we apply it to several cases of interest in this paper.

\section{Entangler}

Given an entangling profile $g(x)$ on the line, we define an entangling profile on the circle $g_{c}(x)$ as

$$
g_{c}(x)=\sum_{n \in \mathbb{Z}} g\left(x+n l_{c}\right) .
$$

It follows from the above theorem that the Fourier transforms $g(k)$ and $g_{c}(n)$ on the line and on the circle

$$
\begin{gathered}
g(k) \equiv \int_{\mathbb{R}} d x e^{-i k x} g(x), \\
g_{c}(n) \equiv \int_{0}^{l_{c}} d x e^{-i k_{n} x} g_{c}(x)
\end{gathered}
$$

are related through

$$
g_{c}(n)=g\left(k_{n}\right)
$$

\section{Two-point correlators}

Next we consider a state $|\Psi\rangle$ on the line characterized by annihilation operators $b(k)$ as

$$
\begin{gathered}
b(k)|\Psi\rangle=0, \quad \forall k \in \mathbb{R}, \\
b(k) \equiv \sqrt{\frac{\beta(k)}{2}} \phi(k)+\frac{i}{\sqrt{2 \beta(k)}} \pi(k) .
\end{gathered}
$$

Its correlation functions in momentum space read

$$
\begin{aligned}
& \left\langle\Psi\left|\phi(k) \phi\left(k^{\prime}\right)\right| \Psi\right\rangle \equiv \delta\left(k+k^{\prime}\right) C_{\phi \phi}(k), \\
& \left\langle\Psi\left|\pi(k) \pi\left(k^{\prime}\right)\right| \Psi\right\rangle \equiv \delta\left(k+k^{\prime}\right) C_{\pi \pi}(k),
\end{aligned}
$$

for

$$
C_{\phi \phi}(k)=\frac{1}{2 \beta(k)}, \quad C_{\pi \pi}(k)=\frac{\beta(k)}{2},
$$

whereas in real space we have

$$
\begin{aligned}
C_{\phi \phi}(x) & \equiv\langle\Psi|\phi(x) \phi(0)| \Psi\rangle=\int_{\mathbb{R}} \frac{d k}{2 \pi} e^{i k x} C_{\phi \phi}(k), \\
C_{\pi \pi}(x) & \equiv\langle\Psi|\pi(x) \pi(0)| \Psi\rangle=\int_{\mathbb{R}} \frac{d k}{2 \pi} e^{i k x} C_{\pi \pi}(k) .
\end{aligned}
$$

Similarly, we consider a state $\left|\Psi_{c}\right\rangle$ on the circle characterized by annihilation operators $b(n)$ as

$$
\begin{gathered}
b(n)\left|\Psi_{c}\right\rangle=0, \quad \forall n \in \mathbb{Z}, \\
b(n) \equiv \sqrt{\frac{\beta_{c}(n)}{2}} \phi(n)+\frac{i}{\sqrt{2 \beta_{c}(n)}} \pi(n),
\end{gathered}
$$

which has correlators

$$
\begin{aligned}
& \left\langle\Psi\left|\phi(n) \phi\left(n^{\prime}\right)\right| \Psi\right\rangle=\delta_{n,-n^{\prime}} C_{c, \phi \phi}(n), \\
& \left\langle\Psi\left|\pi(n) \pi\left(n^{\prime}\right)\right| \Psi\right\rangle=\delta_{n,-n^{\prime}} C_{c, \pi \pi}(n),
\end{aligned}
$$

for

$$
C_{c, \phi \phi}(n)=\frac{1}{2 \beta_{c}(n)}, \quad C_{c, \pi \pi}(n)=\frac{\beta_{c}(n)}{2},
$$

or, in real space,

$$
\begin{aligned}
C_{c, \phi \phi}(x) & \equiv\left\langle\Psi_{c}|\phi(x) \phi(0)| \Psi_{c}\right\rangle \\
& =\frac{1}{l_{c}} \sum_{n \in \mathbb{Z}} e^{i k_{n} x} C_{c, \phi \phi}(n), \\
C_{c, \pi \pi}(x) & \equiv\left\langle\Psi_{c}|\phi(x) \phi(0)| \Psi_{c}\right\rangle \\
& =\frac{1}{l_{c}} \sum_{n \in \mathbb{Z}} e^{i k_{n} x} C_{c, \pi \pi}(n) .
\end{aligned}
$$


Above we did not yet assume any relation between the line annihilation operators $b(k)$ in Eq. (C33) and the circle annihilation operators $b(n)$ in Eq. (C40). Let us now assume that they are related through

$$
\beta_{c}(n)=\beta\left(k_{n}\right) \text {. }
$$

(As reviewed below, this relation naturally holds for ground states of local Hamiltonians. It also holds, by construction, in the cMERA.) It follows that the momentum space correlators on the line and on the circle are related by

$$
C_{c, \phi \phi}(n)=C_{\phi \phi}\left(k_{n}\right), \quad C_{c, \pi \pi}(n)=C_{\pi \pi}\left(k_{n}\right) .
$$

Then the above theorem implies that the real-space correlators on the line and on the circle are related by

$$
\begin{aligned}
& C_{c, \phi \phi}(x)=\sum_{n \in \mathbb{Z}} C_{\phi \phi}\left(x+n l_{c}\right), \\
& C_{c, \pi \pi}(x)=\sum_{n \in \mathbb{Z}} C_{\pi \pi}\left(x+n l_{c}\right) .
\end{aligned}
$$

\section{Ground states of local Hamiltonians}

Let us specialize the above discussion to the ground state of a generic noninteracting local QFT Hamiltonian. Here, we follow closely the analysis of Zou et al. in an Appendix of Ref. [31] on the line, which we (trivially) extend to include also the circle. Let $H^{\mathrm{QFT}}$ denote a Hamiltonian of the form

$$
\begin{aligned}
H^{\mathrm{QFT}} & \equiv \frac{1}{2} \int_{\mathbb{R}} d x \sum_{l}\left\{a_{l}\left[\partial_{x}^{l} \pi(x)\right]^{2}+b_{l}\left[\partial_{x}^{l} \phi(x)\right]^{2}\right\} \\
& =\frac{1}{2} \int_{\mathbb{R}} d k \sum_{l} k^{2 l}\left[a_{l} \pi(-k) \pi(k)+b_{l} \phi(-k) \phi(k)\right] \\
& =\int_{\mathbb{R}} d k E(k) b^{\dagger}(k) b(k),
\end{aligned}
$$

where $E(k)$ is the single-particle energy $E(k)$ and $b(k)$ is an annihilation operator,

$$
b(k) \equiv \sqrt{\frac{\beta(k)}{2}} \phi(k)+\frac{i}{\sqrt{2 \beta(k)}} \pi(k) .
$$

Let us first determine $E(k)$ and $\beta(k)$. Since

$$
\begin{aligned}
& b^{\dagger}(k) b(k)+b^{\dagger}(-k) b(-k) \\
& \quad=\beta(k) \phi(-k) \phi(k)+\frac{1}{\beta(k)} \pi(-k) \pi(k),
\end{aligned}
$$

from requiring

$$
\begin{aligned}
& E(k)\left[b^{\dagger}(k) b(k)+b^{\dagger}(-k) b(-k)\right] \\
& \quad=\sum_{l} k^{2 l}\left[a_{l} \pi(-k) \pi(k)+b_{l} \phi(-k) \phi(k)\right],
\end{aligned}
$$

we conclude that

$$
\begin{array}{r}
E(k) \beta(k)=Q(k), \quad E(k) \frac{1}{\beta(k)}=P(k), \\
\text { for } P(k) \equiv \sum_{l} a_{l} k^{2 l} \text { and } Q(k) \equiv \sum_{l} b_{l} k^{2 l}, \text { or } \\
E(k)=\sqrt{Q(k) P(k)}, \quad \beta(k)=\sqrt{\frac{Q(k)}{P(k)}} .
\end{array}
$$

Let us repeat this calculation for a Hamiltonian $H_{c}^{\mathrm{QFT}}$ obtained from the same Hamiltonian density integrated on the circle, namely,

$$
\begin{aligned}
& H_{c}^{\mathrm{QFT}} \\
& \quad \equiv \frac{1}{2} \int_{0}^{l_{c}} d x \sum_{l}\left\{a_{l}\left[\partial_{x}^{l} \pi(x)\right]^{2}+b_{l}\left[\partial_{x}^{l} \phi(x)\right]^{2}\right\} \\
& =\frac{1}{2} \sum_{n} \sum_{l}\left(k_{n}\right)^{2 l}\left[a_{l} \pi(-n) \pi(n)+b_{l} \phi(-n) \phi(n)\right] \\
& =\sum_{n} E_{c}(n) b^{\dagger}(n) b(n),
\end{aligned}
$$

where

$$
b(n) \equiv \sqrt{\frac{\beta_{c}(n)}{2}} \phi(n)+\frac{i}{\sqrt{2 \beta_{c}(n)}} \pi(n) .
$$

We have

$$
\begin{aligned}
& b^{\dagger}(n) b(n)+b^{\dagger}(-n) b(-n) \\
& \quad=\beta_{c}(n) \phi(-n) \phi(n)+\frac{1}{\beta_{c}(n)} \pi(-n) \pi(n),
\end{aligned}
$$

and, arguing similarly as above, we arrive at

$$
E_{c}(n) \beta_{c}(n)=Q_{c}(n), \quad E_{c}(n) \frac{1}{\beta_{c}(n)}=P_{c}(n)
$$

for $P_{c}(n) \equiv \sum_{l} a_{l}\left(k_{n}\right)^{2 l}$ and $Q_{c}(l) \equiv \sum_{l} b_{l}\left(k_{n}\right)^{2 l}$, or

$$
E_{c}(n)=\sqrt{Q_{c}(n) P_{c}(n)}, \quad \beta_{c}(n)=\sqrt{\frac{Q_{c}(n)}{P_{c}(n)}} .
$$

Finally, we notice that since $Q_{c}(n)=Q\left(k_{n}\right)$ and $P_{c}(n)=$ $P\left(k_{n}\right)$, it follows that $\beta(k)$ on the line and $\beta_{c}(n)$ on the circle are related by $\beta_{c}(n)=\beta\left(k_{n}\right)$, that is, Eq. (C48). Therefore the ground states $\left|\Psi^{\mathrm{QFT}}\right\rangle$ on the line and $\left|\Psi_{c}^{\mathrm{QFT}}\right\rangle$ on the circle, given by

$$
\begin{aligned}
& b(k)\left|\Psi^{\mathrm{QFT}}\right\rangle=0, \quad \forall k \in \mathbb{R}, \\
& b(n)\left|\Psi_{c}^{\mathrm{QFT}}\right\rangle=0, \quad \forall n \in \mathbb{Z},
\end{aligned}
$$

have correlators that are indeed related by Eq. (C50),

$$
\begin{aligned}
& C_{c, \phi \phi}^{\mathrm{QFT}}(x)=\sum_{n \in \mathbb{Z}} C_{\phi \phi}^{\mathrm{QFT}}\left(x+n l_{c}\right), \\
& C_{c, \pi \pi}^{\mathrm{QFT}}(x)=\sum_{n \in \mathbb{Z}} C_{\pi \pi}^{\mathrm{QFT}}\left(x+n l_{c}\right) .
\end{aligned}
$$

\section{CMERA}

On the line, consider an entangler of the form

$$
\tilde{K}(s)=\frac{1}{2} \int_{\mathbb{R}} d k \tilde{g}(s, k)[\pi(-k) \phi(k)+\phi(-k) \pi(k)] .
$$

The cMERA state $\left|\Psi^{\Lambda}(s)\right\rangle$, defined by

$$
\left|\Psi^{\Lambda}(s)\right\rangle \equiv \mathcal{P} \exp \left(-i \int_{0}^{s} d s^{\prime} \tilde{K}\left(s^{\prime}\right)\right)|\Lambda\rangle
$$


is then annihilated by operators $\tilde{b}^{\Lambda}(s, k)$,

$$
\tilde{b}^{\Lambda}(s, k)\left|\Psi^{\Lambda}(s)\right\rangle=0, \quad \forall k \in \mathbb{R},
$$

such that

$$
\tilde{b}^{\Lambda}(s, k) \equiv \sqrt{\frac{\tilde{\beta}(s, k)}{2}} \phi(k)+\frac{i}{\sqrt{2 \tilde{\beta}(s, k)}} \pi(k) .
$$

We saw that $\tilde{\beta}(s, k)$ obeys the differential equation

$$
\frac{\partial}{\partial s} \tilde{\beta}(s, k)=-2 \tilde{g}(s, k) \tilde{\beta}(s, k), \quad \forall k \in \mathbb{R},
$$

with initial condition

$$
\tilde{\beta}(s=0, k)=\Lambda, \quad \forall k \in \mathbb{R} .
$$

On the circle, consider now an entangler of the form

$$
\tilde{K}_{c}(s)=\frac{1}{2} \sum_{n \in \mathbb{N}} \tilde{g}_{c}(s, n)[\pi(-n) \phi(n)+\phi(-n) \pi(n)] .
$$

The cMERA state $\left|\Psi^{\Lambda}(s)\right\rangle$,

$$
\left|\Psi_{c}^{\Lambda}(s)\right\rangle \equiv \mathcal{P} \exp \left(-i \int_{0}^{s} d s^{\prime} \tilde{K}_{c}\left(s^{\prime}\right)\right)\left|\Lambda_{c}\right\rangle,
$$

is annihilated by operators $b^{\Lambda}(s, n)$,

$$
\begin{gathered}
\tilde{b}^{\Lambda}(s, n)\left|\Psi_{c}^{\Lambda}(s)\right\rangle=0, \quad \forall n \in \mathbb{Z}, \\
\tilde{b}^{\Lambda}(s, n) \equiv \sqrt{\frac{\tilde{\beta}_{c}(s, n)}{2}} \phi(n)+\frac{i}{\sqrt{2 \tilde{\beta}_{c}(s, k)}} \pi(n),
\end{gathered}
$$

where $\beta_{c}(s, n)$ obeys the differential equation

$$
\frac{\partial}{\partial s} \tilde{\beta}_{c}(s, n)=-2 \tilde{g}_{c}(s, n) \tilde{\beta}_{c}(s, n), \quad \forall n \in \mathbb{Z},
$$

with the initial condition

$$
\tilde{\beta}_{c}(s=0, n)=\Lambda, \quad \forall n \in \mathbb{Z} .
$$

Clearly, Eqs. (C73) and (C74) for $\tilde{\beta}(s, k)$ on the line and Eqs. (C79) and (C80) for $\tilde{\beta}_{c}(s, n)$ on the circle are very similar. If, following the proposal of this work [see Eq. (21) in the main text], we set the entangling profile on the line and on the circle to be related by Eq. (C31), that is,

$$
\tilde{g}_{c}(s, n)=\tilde{g}\left(s, k_{n}\right),
$$

then Eqs. (C73), (C74), (C79), and (C80) imply that also

$$
\tilde{\beta}_{c}(s, n)=\tilde{\beta}\left(s, k_{n}\right), \quad \forall n \in \mathbb{Z} .
$$

This last equation is analogous to condition (C48). Therefore the correlators of the cMERA state on the line and on the circle are indeed related by Eq. (C50). That is,

$$
\begin{aligned}
& C_{c, \phi \phi}^{\Lambda}(s, x)=\sum_{n \in \mathbb{Z}} C_{\phi \phi}^{\Lambda}\left(s, x+n l_{c}\right), \\
& C_{c, \pi \pi}^{\Lambda}(s, x)=\sum_{n \in \mathbb{Z}} C_{\pi \pi}^{\Lambda}\left(s, x+n l_{c}\right) .
\end{aligned}
$$

\section{APPENDIX D: GENERALIZATIONS BEYOND THE CIRCLE}

In this last Appendix we briefly comment on possible generalizations of the results presented in this paper, based on applying the method of images to other geometries.

In its essence, the method of images is based on generating solutions of a linear differential equation (the equations of motion) by summing over different solutions related to each other by a discrete symmetry transformation (such as a translation in the line by a distance $l_{c}$, which allows us to turn the line into a circle of size $l_{c}$ ). This is possible for linear systems, and thus free theories. The same techniques thus admit immediate generalizations.

\section{Higher dimensions}

One generalization is to obtain the cMERA on an $\mathrm{N}$ dimensional torus $T^{N}$ from $\mathbb{R}^{N}$, since an $N$-torus can be thought of as an orbifold of $\mathbb{R}^{N}$.

This is done by repeating the computation, with a sum over images in $N$ directions separately. To be precise, on an $N$ torus, a field $\phi(x)$ is expressible as

$$
\phi(x)=\sum_{\left\{n_{i}\right\}}\left(\prod_{i}^{N} \frac{1}{\sqrt{l_{c_{i}}}}\right) e^{i k_{\left.\mid n_{i}\right\}} \cdot x} \phi\left(\left\{n_{i}\right\}\right),
$$

where $x$ is an $N$-component vector, $k_{\left\{n_{i}\right\}}$ is also an $N$ component vector given by

$$
k_{\left\{n_{i}\right\}}=\left\{\frac{2 \pi n_{i}}{l_{c_{i}}}\right\}, \quad n_{i} \in \mathbb{Z},
$$

and $l_{c_{i}}$ is the periodicity of each cycle $i$ in $T^{N}$. A similar expression applies to $\pi(x)$. The cMERA entangler would take the form

$$
\begin{aligned}
K_{T^{N}}(s) & \\
= & \frac{1}{2} \int_{T^{N}} d^{N} x d^{N} y g_{T^{N}}(s, x-y) \\
& \times[\pi(x) \phi(y)+\phi(x) \pi(y)] \\
= & \frac{1}{2} \sum_{\left\{n_{i} \in \mathbb{N}\right\}} g_{T^{N}}\left(s,\left\{n_{i}\right\}\right)\left[\pi\left(\left\{-n_{i}\right\}\right) \phi\left(\left\{n_{i}\right\}\right)\right. \\
& \left.+\pi\left(\left\{-n_{i}\right\}\right) \phi\left(\left\{n_{i}\right\}\right)\right] .
\end{aligned}
$$

Here, $g_{T^{N}}\left(s,\left\{n_{i}\right\}\right)$ again correspond to the Fourier transform of $g_{T^{N}}(s, x-y)$. The method of images would imply that one can generate an admissible entangler on $T^{N}$ using the entangler on the $\mathbb{R}^{N}$, i.e.,

$$
g_{T^{N}}(s, x)=\sum_{\left\{n_{i}\right\}} g_{\mathbb{R}^{N}}\left(s, x_{\left\{n_{i}\right\}}\right), \quad x_{\left\{n_{i}\right\}} \equiv\left\{x_{i}+n_{i} l_{c_{i}}\right\} .
$$

On $T^{2}$, for example, this procedure generates doubly periodic functions. The method of images is widely applied in two-dimensional CFT (CFT2) to generate correlation functions on $T^{2}$ in minimal models, which admit free-field representations. 


\section{Open boundaries}

One can also generate systems with boundaries using the method of images. Consider for concreteness a onedimensional quantum system on the half line. That is, there is a boundary at $x=0$. The effect of the boundary can be treated as a mirror, in which inserting an operator $\mathcal{O}(x, t)$ in the presence of the boundary is equivalent to inserting a pair of operators $\mathcal{O}(x, t)$ and $\mathcal{O}(-x, t)$ in the theory which is defined on the real line with no boundaries. In complex coordinates $z=t+i x$ and $\bar{z}=t-i x$, this gives

$$
\langle\mathcal{O}(z, \bar{z}) \cdots\rangle_{\mathrm{UHP}}=\left\langle\mathcal{O}(z) \overline{\mathcal{O}}^{P}(z *) \cdots\right\rangle_{\mathbb{R}^{2}},
$$

where $\mathcal{O}^{P}(z *)$ corresponds to the parity-transformed antiholomorphic part of the operator $\mathcal{O}(z, \bar{z})$ and UHP refers to the upper half plane. The parity transformation turns the antiholomorphic operator into a holomorphic one, now inserted at $z *$. As an example of such a parity transformation, consider a free bosonic field $\phi(z)$. Then

$$
\left(\partial_{\bar{z}} \bar{\phi}\right)^{P}(z *) \equiv \eta_{P} \partial_{z} \phi(z *),
$$

where $\eta_{P}= \pm 1$, depending on the precise boundary condition that is imposed. For example, the Dirichlet boundary condition that sets $\phi(z, \bar{z})=0$ at the boundary located on the real line gives $\eta_{P}=1$, whereas the Neumann boundary condition that sets the derivative of $\phi$ to zero gives $\eta_{P}=-1$. We note that in this context of conformal boundary conditions, the method of images encapsulated in (D6) works beyond freefield theories because in two-dimensional CFTs holomorphic and antiholomorphic conformal transformations are independent.

This fact thus allows one to generate cMERA entanglers applicable to theories with boundaries from entanglers on the real line using the method of images. Consider the specific case of a free boson $\phi(x)$ again.

Suppose we impose the Neumann boundary condition at $x=0$,

$$
\partial_{x} \phi(0)=0 .
$$

The mode expansion of $\phi_{\mathbb{R}>0}(x)$ and $\pi_{\mathbb{R}>0}(x)$ would then be given by

$$
\begin{gathered}
\phi_{\mathbb{R}>0}(x)=\frac{2}{\sqrt{2 \pi}} \int_{0}^{\infty} d k \cos (k x) \phi_{\mathbb{R}>0}(k), \\
\pi_{\mathbb{R}>0}(x)=\frac{2}{\sqrt{2 \pi}} \int_{0}^{\infty} d k \cos (k x) \pi_{\mathbb{R}>0}(k) .
\end{gathered}
$$

We note that this implies that

$$
\phi_{\mathbb{R}>0}(x)=\frac{1}{2}\left[\phi_{\mathbb{R}}(x)+\phi_{\mathbb{R}>0}(-x)\right]
$$

and

$$
\phi_{\mathbb{R}>0}(k)=\frac{1}{2}\left[\phi_{\mathbb{R}}(k)+\phi_{\mathbb{R}}(-k)\right]
$$

and similarly for $\pi_{\mathbb{R}>0}(x)$ and its Fourier modes. In other words, we have selected a boundary condition that imposes that the Fourier modes are even in $k$.

Consider the entangler characterized by the function $g(s, x)$ discussed in the main text. On the half line this is given by

$$
\begin{aligned}
\tilde{K}_{\mathbb{R}>0}(s)= & \frac{1}{2} \int_{\mathbb{R}^{2}>0} d x d y \tilde{g}_{\mathbb{R}>0}(x, y)\left(\phi_{\mathbb{R}>0}(x) \pi_{\mathbb{R}>0}(y)\right. \\
& \left.+\pi_{\mathbb{R}>0}(x) \phi_{\mathbb{R}>0}(y)\right) .
\end{aligned}
$$

Now using (D11), we notice that this can be unfolded into an integral over $\mathbb{R}^{2}$,

$$
\tilde{K}_{\mathbb{R}>0}(s)=\frac{1}{2} \int_{\mathbb{R}^{2}} d x d y \tilde{g}(x, y)[\phi(x) \pi(y)+\pi(x) \phi(y)],
$$

if we define

$$
\tilde{g}(-|x|,|y|)=\tilde{g}(|x|,-|y|)=\tilde{g}(-|x|,-|y|)=\tilde{g}(|x|,|y|) .
$$

This implies that the desired solution of an entangler on the half line can be generated from that of the entangler on the real line $\tilde{g}_{\mathbb{R}}(x-y)$ by

$$
\begin{aligned}
\tilde{g}_{\mathbb{R}>0}(x, y)= & \frac{1}{4}\left[\tilde{g}_{\mathbb{R}}(x-y)+\tilde{g}_{\mathbb{R}}(-x-y)\right. \\
& \left.+\tilde{g}_{\mathbb{R}}(x+y)+\tilde{g}_{\mathbb{R}}(-x-y)\right] .
\end{aligned}
$$

In general beyond free-field theories, the entanglers admit generalizations. In the perturbative limit we expect that the method of images can be generalized. Let us briefly comment on that. In an interacting theory, the ansatz for the disentangler would generally involve nonlinear terms. This has been considered for example in Refs. [25-28]. As an example it is possible that a cubic term could feature in the ansatz

$$
\begin{aligned}
K_{3}= & \int d x_{1} d x_{2} d x_{3} g^{a b c}\left(s,, x_{1}, x_{2}, x_{3}\right) \phi^{a}\left(x_{1}\right) \\
& \times \phi^{b}\left(x_{2}\right) \phi^{c}\left(x_{3}\right)+\cdots,
\end{aligned}
$$

where $\phi^{1}(x) \equiv \phi(x)$ and $\phi^{2}(x) \equiv \pi(x)$, while $\cdots$ refers to a sum that includes all Hermitian conjugates to make $K_{3}$ Hermitian. Generically, the field theory enjoys translation invariance. Therefore $g_{c}^{a b c}\left(s, x_{1}, x_{2}, x_{3}\right)$ is expected to depend only on the difference between $x_{1}, x_{2}, x_{3}$. That is, $g^{a b c}\left(s, x_{1}, x_{2}, x_{3}\right) \equiv \tilde{g}^{a b c}\left(s, \Delta_{12}, \Delta_{23}\right)$, where $\Delta_{i j}=x_{j}-$ $x_{i}$. The disentangler on a circle can then be obtained via

$$
\tilde{g}_{c}^{a b c}(s, x, y)=\sum_{n, m \in \mathbb{Z}} \tilde{g}^{a b c}\left(s, x+n l_{c}, y+m l_{c}\right) .
$$

In momentum space,

$$
K_{3 c}=\sum_{m, n \in \mathbb{Z}} g^{a b c}(s, m, n) \phi_{m}^{a} \phi_{n}^{b} \phi_{-m-n}^{c}+\cdots,
$$

where

$$
\tilde{g}_{c}^{a b c}(s, m, n) \equiv \tilde{g}_{3}^{a b c}\left(s, k_{m}, k_{n}\right),
$$

analogous to (22).

We note, however, that it is known that in large- $N$ theories, such as CFTs with an AdS holographic dual, correlation functions at finite temperatures can be obtained from those at zero temperatures by the method of images - at least for sufficiently low temperatures [46]. Therefore there are hopes that the method of images that maps cMERA to other cMERAs defined on a compact space can be applied even if the theory concerned is interacting. 
[1] G. Vidal, Entanglement Renormalization, Phys. Rev. Lett. 99, 220405 (2007).

[2] G. Vidal, A Class of Quantum Many-Body States That Can Be Efficiently Simulated, Phys. Rev. Lett. 101, 110501 (2008).

[3] G. Evenbly and G. Vidal, Algorithms for entanglement renormalization, Phys. Rev. B 79, 144108 (2009).

[4] M. Aguado and G. Vidal, Entanglement Renormalization and Topological Order, Phys. Rev. Lett. 100, 070404 (2008).

[5] V. Giovannetti, S. Montangero, and R. Fazio, Quantum Multiscale Entanglement Renormalization Ansatz Channels, Phys. Rev. Lett. 101, 180503 (2008).

[6] R. N. C. Pfeifer, G. Evenbly, and G. Vidal, Entanglement renormalization, scale invariance, and quantum criticality, Phys. Rev. A 79, 040301(R) (2009).

[7] G. Evenbly and G. Vidal, Quantum criticality with the multi-scale entanglement renormalization ansatz, in Strongly Correlated Systems: Numerical Methods, Springer Series in Solid-State Sciences Vol. 176 (Springer, Berlin, 2013), Chap. 4, pp. 99-130.

[8] G. Evenbly and G. Vidal, Theory of minimal updates in holography, Phys. Rev. B 91, 205119 (2015).

[9] G. Evenbly and G. Vidal, Tensor Network Renormalization Yields the Multi-scale Entanglement Renormalization Ansatz, Phys. Rev. Lett. 115, 200401 (2015).

[10] B. Swingle, Entanglement renormalization and holography, Phys. Rev. D 86, 065007 (2012).

[11] B. Swingle, Constructing holographic spacetimes using entanglement renormalization, arXiv:1209.3304 [hep-th].

[12] B. Czech, L. Lamprou, S. McCandlish, and J. Sully, Tensor networks from kinematic space, J. High Energy Phys. 07 (2016) 100.

[13] A. Milsted and G. Vidal, Geometric interpretation of the multiscale entanglement renormalization ansatz, arXiv:1812.00529 [hep-th].

[14] N. A. McMahon, S. Singh, and G. K. Brennen, A holographic duality from lifted tensor networks, npj Quantum Inf. 6, 36 (2020).

[15] C. Beny, Causal structure of the entanglement renormalization ansatz, New J. Phys. 15, 023020 (2013).

[16] R. S. Kunkolienkar and K. Banerjee, Towards a dS/MERA correspondence, Int. J. Mod. Phys. D 26, 1750143 (2017).

[17] N. Bao, C. J. Cao, S. M. Carroll, and A. Chatwin-Davies, de Sitter space as a tensor network: Cosmic no-hair, complementarity, and complexity, Phys. Rev. D 96, 123536 (2017).

[18] A. J. Ferris and D. Poulin, Tensor Networks and Quantum Error Correction, Phys. Rev. Lett. 113, 030501 (2014).

[19] A. J. Ferris and D. Poulin, Branching MERA codes: A natural extension of classical and quantum polar codes, in 2014 IEEE International Symposium on Information Theory (ISIT) (IEEE, Piscataway, 2014), pp. 1081-1085.

[20] C. Bény, Deep learning and the renormalization group, arXiv:1301.3124 [quant-ph].

[21] E. M. Stoudenmire and D. J. Schwab, Supervised learning with quantum-inspired tensor networks, Adv. Neural Inf. Process. Syst. 29, 4799 (2016).

[22] Y. Levine, O. Sharir, N. Cohen, and A. Shashua, Quantum Entanglement in Deep Learning Architectures, Phys. Rev. Lett. 122, 065301 (2019).

[23] I. Cong, S. Choi, and M. D. Lukin, Quantum convolutional neural networks, Nat. Phys. 15, 1273 (2019).
[24] J. Haegeman, T. J. Osborne, H. Verschelde, and F. Verstraete, Entanglement Renormalization for Quantum Fields in Real Space, Phys. Rev. Lett. 110, 100402 (2013)

[25] J. S. Cotler, J. Molina-Vilaplana, and M. T. Mueller, A Gaussian variational approach to cMERA for interacting fields, arXiv:1612.02427 [hep-th].

[26] J. S. Cotler, M. Reza Mohammadi Mozaffar, A. Mollabashi, and A. Naseh, Entanglement renormalization for weakly interacting fields, Phys. Rev. D 99, 085005 (2019).

[27] J. J. Fernandez-Melgarejo, J. Molina-Vilaplana, and E. Torrente-Lujan, Entanglement renormalization for interacting field theories, Phys. Rev. D 100, 065025 (2019).

[28] J. J. Fernández-Melgarejo and J. Molina-Vilaplana, NonGaussian entanglement renormalization for quantum fields, J. High Energy Phys. 07 (2020) 149.

[29] Q. Hu and G. Vidal, Spacetime Symmetries and Conformal Data in the Continuous Multiscale Entanglement Renormalization Ansatz, Phys. Rev. Lett. 119, 010603 (2017).

[30] A. Franco-Rubio and G. Vidal, Entanglement and correlations in the continuous multi-scale entanglement renormalization ansatz, J. High Energy Phys. 12 (2017) 129.

[31] Y. Zou, M. Ganahl, and G. Vidal, Magic entanglement renormalization for quantum fields, arXiv:1906.04218 [condmat.str-el].

[32] A. Franco-Rubio and G. Vidal, Entanglement renormalization for gauge invariant quantum fields, Phys. Rev. D 103, 025013 (2021).

[33] A. Mollabashi, M. Naozaki, S. Ryu, and T. Takayanagi, Holographic geometry of cMERA for quantum quenches and finite temperature, J. High Energy Phys. 03 (2014) 098.

[34] M. Nozaki, S. Ryu, and T. Takayanagi, Holographic geometry of entanglement renormalization in quantum field theories, J. High Energy Phys. 10 (2012) 193.

[35] J. Molina-Vilaplana, Information geometry of entanglement renormalization for free quantum fields, J. High Energy Phys. 09 (2015) 002.

[36] J. Molina-Vilaplana, Entanglement renormalization and two dimensional string theory, Phys. Lett. B 755, 421 (2016).

[37] M. Miyaji, S. Ryu, T. Takayanagi, and X. Wen, Boundary states as holographic duals of trivial spacetimes, J. High Energy Phys. 05 (2015) 152.

[38] M. Miyaji, T. Numasawa, N. Shiba, T. Takayanagi, and K. Watanabe, cMERA as Surface/State Correspondence in AdS/CFT, Phys. Rev. Lett. 115, 171602 (2015).

[39] M. Miyaji and T. Takayanagi, Surface/state correspondence as a generalized holography, Prog. Theor. Exp. Phys. 2015, 073 B03 (2015).

[40] X. Wen, G. Y. Cho, P. L. S. Lopes, Y. Gu, X. L. Qi, and S. Ryu, Holographic entanglement renormalization of topological insulators, Phys. Rev. B 94, 075124 (2016).

[41] A. Franco-Rubio, Entanglement renormalization for quantum fields with boundaries and defects, Phys. Rev. B 104, 125131 (2021).

[42] Reference [24] introduced a more general class of scaledependent entangler $K(s)$, which nevertheless maintained a constant entangling scale $1 / \Lambda$. For simplicity, in this paper we restrict our attention to scale-independent entanglers $K \neq$ $K(s)$ [which then become scale-dependent entanglers $\tilde{K}(s) \equiv$ $e^{i s D}(L+K-D) e^{-i s D}$ in the rescaled picture used in this paper]. A generalization of our results to a scale-dependent entangler 
$K(s)$ requires more complex notation but is otherwise straightforward.

[43] In the usual picture of cMERA, the UV and IR lengths are given by $1 / \Lambda$ and $e^{s} / \Lambda$, respectively. In the rescaled picture used in this paper, the UV and IR lengths are instead given by $e^{-s} / \Lambda$ and $\Lambda$.

[44] Correlation functions on a circle cannot be obtained from correlation functions on a line by the method of images in a generic interacting theory. In strongly interacting theories, it takes some special requirements, such as the large- $N$ limit in matrix theories, for the method of images to work well. Please refer to Ref. [46] for details.

[45] B. Czech, G. Evenbly, L. Lamprou, S. McCandlish, X. L. Qi, J. Sully, and G. Vidal, Tensor network quotient takes the vacuum to the thermal state, Phys. Rev. B 94, 085101 (2016).

[46] M. Brigante, Aspects of finite temperature field theories in AdS/CFT, Ph.D. thesis, Massachusetts Institute of Technology, 2008. 